\title{
Background errors in a global wave model determined from altimeter data
}

\author{
Diana J. M. Greenslade \\ Bureau of Meteorology Research Centre, Melbourne, Victoria, Australia
}

Ian R. Young

Swinburne University of Technology, Melbourne, Victoria, Australia

Received 12 February 2004; revised 1 June 2004; accepted 2 July 2004; published 18 September 2004.

[1] One of the main limitations to current wave data assimilation systems is the lack of an accurate representation of the structure of the background errors. One method that may be used to determine background errors is the observational method of Hollingsworth and Lönnberg [1986]. This method considers correlations of the differences between observations and the background. For the case of Significant Wave Height (SWH), potential observations come from satellite altimeters. In this paper, correlations of the differences between modeled SWH and bias-corrected ERS-2 data are calculated. The irregular sampling pattern of the altimeter is accounted for by adjusting the correlation length scales according to latitude and the calculated length scale. The results show that the length scale of the background errors varies significantly over the globe, with the largest scales at low latitudes and shortest scales at high latitudes. Very little seasonal or year-to-year variability in the correlation length scales is detected. Conversely, the magnitude of the background error variance is found to have considerable seasonal and year-to-year variability. By separating the altimeter ground tracks into ascending and descending tracks, it is possible to examine, to a limited extent, whether any anisotropy exists in the background errors. INDEX TERMS: 4247 Oceanography: General: Marine meteorology; 3337 Meteorology and Atmospheric Dynamics: Numerical modeling and data assimilation; 4560 Oceanography: Physical: Surface waves and tides (1255); KEYWORDS: waves, data assimilation, background errors

Citation: Greenslade, D. J. M., and I. R. Young (2004), Background errors in a global wave model determined from altimeter data, J. Geophys. Res., 109, C09007, doi:10.1029/2004JC002324.

\section{Introduction}

[2] Several operational Numerical Weather Prediction (NWP) centers around the world run wave forecasting systems that routinely assimilate satellite SWH data or, more recently, directional wave spectra. There has been a considerable amount of research into the development of wave data assimilation systems in recent years. A major limitation to current assimilation systems is the specification of the model (or background) errors. This has not previously been explored to any great extent for wave models.

[3] One method commonly used to investigate the background errors is the observational method of Hollingsworth and Lönnberg [1986]. This method considers correlations of the differences between observations from a dense, longterm observational network and the background field. In this work, global observations of SWH from the ERS-2 satellite altimeter are compared to SWH from a global wave model to examine the structure of the background errors, and how they vary in space and time.

Copyright 2004 by the American Geophysical Union. 0148-0227/04/2004JC002324
[4] A brief review of previous wave data assimilation research is given in section 2 with an emphasis on specification of the wave model background errors. The wave model used in this work is a version of the WAM model [WAMDI Group, 1988; Komen et al., 1994]. This is described in section 3, and in section 4 the altimeter data are described. The method used to compute the background error correlations is presented in section 5. This includes the derivation of an appropriate correction to account for the irregular altimeter sampling pattern. Results for isotropic and anisotropic correlations are presented and discussed in section 6, and finally, section 7 contains a summary and outlook.

\section{Background}

[5] Current operational wave data assimilation systems at, for example, the European Centre for Medium-Range Weather Forecasts (ECMWF), the Australian Bureau of Meteorology (the Bureau), and Meteo-France use the sequential method of Statistical Interpolation (SI) to combine first-guess wave model fields with the observations to obtain analyzed wave fields. Lionello et al. [1992] were the 
first authors to document SI as a method to construct analyzed SWH fields, although it had been in use in NWP previously [Lorenc, 1981]. The SI algorithm is described here.

[6] At each model gridpoint, $i$, the analyzed (corrected) $\mathrm{SWH}, H_{a n}^{i}$, is expressed as a linear combination of the firstguess predictions from the model, $H_{p}^{i}$, and the observations, $H_{o b s}^{j}$,

$$
H_{a n}^{i}=H_{p}^{i}+\sigma_{p}^{i} \sum_{j=1}^{N_{o b s}} W_{i j}\left[\frac{H_{o b s}^{j}-H_{p}^{j}}{\sigma_{p}^{j}}\right] .
$$

$N_{o b s}$ is the number of observations and $\sigma_{p}$ is the model prediction root-mean-square (rms) error, i.e.,

$$
\sigma_{p}^{i}=\sqrt{\left\langle\left(H_{p}^{i}-T^{i}\right)^{2}\right\rangle},
$$

where $T$ is the true SWH and $\langle\ldots\rangle$ is the expected value. The weights $\left(W_{i j}\right)$ are chosen to minimize the rms error of the analysis. Assuming that the errors in the model are not correlated with the measurement errors, the weights are therefore given by

$$
W_{i j}=\sum_{k=1}^{N_{o b s}} P_{i k} M_{k j}^{-1}
$$

where the matrix $M$ is

$$
M_{k j}=P_{k j}+O_{k j}
$$

$P$ and $O$ are called the error correlation matrices of the model prediction (or background) and observations, respectively, and must be specified. They are symmetric $N_{o b s} \times$ $N_{\text {obs }}$ matrices where the element $(k, j)$ of matrix $P$ is given by

$$
P_{k j}=\frac{\left\langle\left(H_{p}^{k}-T^{k}\right)\left(H_{p}^{j}-T^{j}\right)\right\rangle}{\sigma_{p}^{k} \sigma_{p}^{j}} .
$$

In other words, the value of element $(k, j)$ of matrix $P$ is the correlation between the model error at observation location $k$ and the model error at observation location $j$. Similarly element $(k, j)$ of matrix $O$ is given by

$$
O_{k j}=\frac{\left\langle\left(H_{o b s}^{k}-T^{k}\right)\left(H_{o b s}^{j}-T^{j}\right)\right\rangle}{\sigma_{p}^{k} \sigma_{p}^{j}} .
$$

[7] If $P$ and $O$ are known precisely, then the weights are optimal and $W_{i j}$ does indeed minimize the rms error of the analysis. In that case, the technique is known as Optimal (or Optimum) Interpolation. However, in practice, $P$ and $O$ are not known exactly as they represent the difference between the model (or observation) and the unknown truth. For this reason, the term SI is used here.

[8] Most current operational wave data assimilation schemes use observations from satellite altimeters. The observational errors for altimeter SWH data are generally

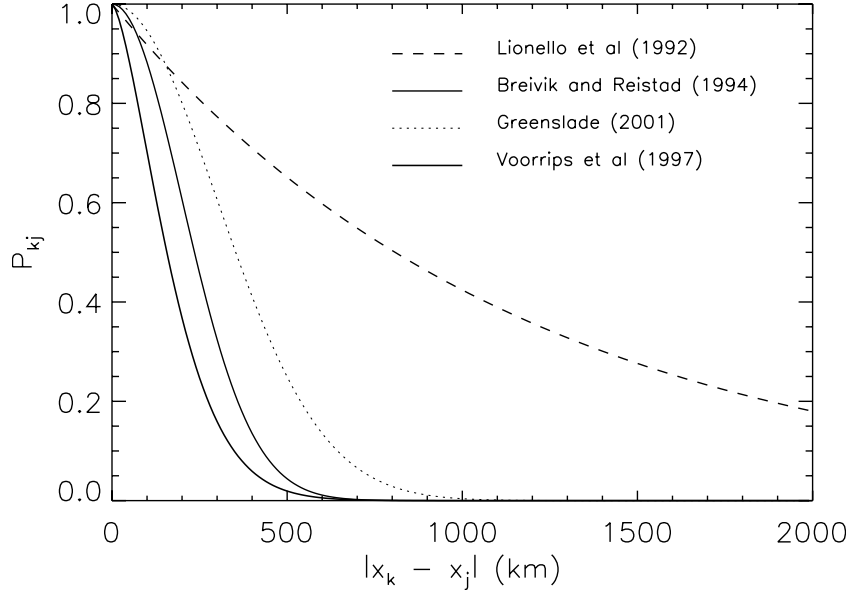

Figure 1. Some example functions used for the background error correlations at midlatitudes.

assumed to be spatially uncorrelated, and therefore $O$ reduces to a diagonal matrix with values of

$$
O_{k k}=\frac{\left\langle\left(H_{o b s}^{k}-T^{k}\right)^{2}\right\rangle}{\sigma_{p}^{k} \sigma_{p}^{k}}=\frac{\sigma_{o b s}^{2}}{\sigma_{p}^{2}}
$$

along the diagonal. Recent work [Janssen et al., 2003] has shown that in fact there may be some correlation between the errors of consecutive along-track altimeter observations. The potential impact of this on the OI scheme would be to increase the lower limit of the expected analysis error [Daley, 1991]. In practice, this could be reduced by alongtrack averaging of the altimeter data or by subsampling the observations.

[9] $P_{k j}$, on the other hand, is known to be horizontally correlated and is more difficult to specify. Lionello et al. [1992] set $P_{k j}$ to be isotropic and homogenous and to have the form

$$
P_{k j}=\exp \left[-\frac{\left|x_{k}-x_{j}\right|}{L}\right]
$$

where $L$ is the decorrelation length scale and $\left|x_{k}-x_{j}\right|$ is the distance between the points $k$ and $j$. Several values were explored for $L$ ranging from 1 to 10 model gridpoints, where the model resolution was $3^{\circ}$. Model forecasts generally improved as $L$ increased, but tended to saturate for $L>15^{\circ}$. $P_{k j}$ for $L=15^{\circ}$ at a latitude of $45^{\circ} \mathrm{N}$ is shown in Figure 1, along with some $P_{k j}$ s proposed by other authors (see below).

[10] This method has been applied in several regional studies. Mastenbroek et al. [1994] assimilated ERS-1 SWH into the WAM model over the North and Norwegian Seas. In this study, the shape of the background error correlations was described with an auto-regressive function, as opposed to the exponential function used by Lionello et al. [1992], so that

$$
P_{k j}=\left(1+\frac{\left|x_{k}-x_{j}\right|}{L}\right) \exp \left[-\frac{\left|x_{k}-x_{j}\right|}{L}\right] .
$$


$L$ was set to be 1.8 model gridpoints, where the spatial resolution of the model was approximately $75 \mathrm{~km}$. Another regional study based on this technique to update the wave spectrum is described by Breivik and Reistad [1994], where ERS-1 SWH was assimilated into a second-generation wave model over the North, Norwegian, and Barents Seas. A successive corrections method was used and the background error correlations were defined as

$$
P_{k j}=\exp \left[-\frac{1}{2} \frac{\left|x_{k}-x_{j}\right|^{2}}{L^{2}}\right] .
$$

Breivik and Reistad [1994] suggested that $L$ would be of similar scale to that of the wind fields in the atmospheric model, so it was set to be $200 \mathrm{~km}$, decreasing to $40 \mathrm{~km}$ over five successive iterations. This background error correlation function is shown (for the initial 200-km value) in Figure 1.

[11] Young and Glowacki [1996] applied an SI method to assimilate GEOSAT data into a second-generation wave model over the Tasman Sea. The background errors were defined to be the same as in equation (10) with the length scale set to $350 \mathrm{~km}$. Bender and Glowacki [1996] applied the method of Lionello et al. [1992] to a version of WAM. Background errors were defined as in equation (8) with $L=$ $350 \mathrm{~km}$. Greenslade [2001] extended this work and performed a similar experiment over the Australian region. $P_{k j}$ was defined as in equation (10). Values of $300 \mathrm{~km}, 500 \mathrm{~km}$, and $700 \mathrm{~km}$ were tested for $L$, and results were evaluated at a number of buoys around the Australian coast. $L=300 \mathrm{~km}$ provided the best results in this study and this structure for $P_{k j}$ is shown in Figure 1.

[12] A sequential method to assimilate directional wave information is described by Hasselmann et al. [1997]. The technique was applied to ERS-1 SAR data in the North Atlantic. The length scale used was $200 \mathrm{~km}$, to allow for the smaller correlation scales of the intense low-pressure systems with which the authors were most concerned. Wind field corrections obtained from the algorithm of Lionello et al. [1992] were combined with the first-guess wind fields via SI to produce analyzed wind fields. The correlation scale for this step was reduced to $50 \mathrm{~km}$ and only applied to neighboring gridpoints.

[13] Voorrips et al. [1997] adapted the method and assimilated wave spectra from pitch-and-roll buoys into WAM over the North Sea. The spatial correlation structure of the background error for total wave energy $\left(E=S W H^{2} / 16\right)$ was estimated by considering differences between the model forecast at 24 hours and the model analysis over a 2-month period. The most appropriate correlation structure was found to be

$$
P_{k j}=\exp \left[-\left(\frac{\left|x_{k}-x_{j}\right|}{L}\right)^{\frac{3}{2}}\right],
$$

with $L=200 \mathrm{~km}$. This is shown in Figure 1. Dunlap et al. [1998] assimilated ERS-1 SAR wave spectra into WAM over the northwest Atlantic. SI was not used, but the observations were spread over a region-of-influence, $R$, around the observation point with a weighting factor,

$$
\mathrm{w}=e^{-R} \quad R=\frac{1}{\sqrt{2}} \frac{r}{L},
$$

where $r$ is the distance from the observation to the model gridpoint and $L$ is a length scale. It was argued that two distinct length scales should be used: a shorter one corresponding to wind-sea systems and a longer one for swell. A compromise of $L=500 \mathrm{~km}$ was used.

[14] Breivik et al. [1998] performed a study assimilating SAR spectra into a second-generation wave model over the North and Norwegian Seas. The successive corrections method of Breivik and Reistad [1994] was used. Since full wave spectra were assimilated, the background error correlation function was defined as a function of wave frequency, $f$,

$$
P_{k j}(f)=\exp \left[-\frac{1}{2} \frac{\left|x_{k}-x_{j}\right|^{2}}{L(f)^{2}}\right] .
$$

Breivik et al. [1998] determined $L$ for SWH empirically by considering colocated model and altimeter SWH estimates over a 4-month period. The domain considered covered the North, Norwegian, and Barents Seas and the west Atlantic. The length scale for SWH was found to be $200-250 \mathrm{~km}$. It was then assumed that the spatial scale of errors in the energy spectra was similar to that of the errors in SWH. In addition, the length scale was defined so that long-wave information from the observations influenced a larger area of the analyzed field than shorter waves. $L(f)$ was thus defined to range from $300 \mathrm{~km}$ for $f=0.04 \mathrm{~Hz}$ to $60 \mathrm{~km}$ for $f=0.24 \mathrm{~Hz}$.

[15] In the studies described here a wide range of structures has been used to represent the background errors. Just a few are shown in Figure 1. Some of these have been ad hoc estimates, while others have provided some justification for the choice of background error structure. Most of the studies have assumed isotropy and homogeneity in the background error structure. To date, there has been no extensive effort made to determine the spatial scale of the background errors in wave models on a global basis. A major limitation to current implementations of SI systems and indeed potential future systems is the lack of an accurate representation of the model or background errors.

[16] The background error is, by definition, the difference between the background and the truth. Of course, the truth is not known, and so other methods to calculate background error must be sought. There are several methods that have commonly been used to estimate the background error correlations in meteorology and oceanography. One of these is the observational method of Hollingsworth and Lönnberg [1986]. This method uses observations from a long-term, dense, homogenous observational network and examines the difference between the observations and the background field. The total error can be partitioned into background error and observational error by assuming that the observational errors are spatially uncorrelated. This method has been used extensively in meteorology. In particular, many studies have used the radiosonde networks that exist over the continental land masses [e.g., Seaman, 1982; Hollingsworth and Lönnberg, 1986; Lönnberg and Hollingsworth, 1986; Thiébaux et al., 1986]. The observational method has the merit of being a technique that calculates background errors directly, and it can be used to determine both the background error variance and its spatial structure.

[17] Highest quality wave observations generally come from buoys or platforms which are fixed in space. However, 
the spatial distribution of buoys is extremely poor: For practical reasons, they are generally located in coastal regions and so it is difficult to obtain any information on the spatial error correlation structure from buoy data. This lack of a long-term observational network with reasonable space-time sampling characteristics over the ocean means that it has been difficult in the past to apply the observational method within the fields of oceanography or marine meteorology. However, satellite altimeters now provide a comprehensive global long-term network of wave observations which can potentially be used to determine the correlation structure of the background errors in a wave model.

[18] One disadvantage of the observational method is that it can only provide information on the spatial scales of the observational network, and in many cases, only over limited areas. This is not a major issue in this work because the satellite altimeter estimates of SWH that are used are closely spaced together in the along-track direction, compared to the spatial scales that are of interest. The issue of the large spacing between altimeter ground tracks and the limited range of directions covered by the ground tracks has been addressed by D. J. M. Greenslade and I. R. Young (The impact of altimeter sampling patterns on estimates of background errors in a global wave model, submitted to Journal of Atmospheric and Oceanic Technology, 2004) (hereinafter referred to as GY1). Results from GY1 will be used in this paper.

\section{Wave Model}

[19] The wave model used in this work is AUSWAM [National Meteorological Operations Centre (NMOC), 1999], a version of the WAM model. The WAM model is a third-generation wave model which solves the wave transport equation explicitly without assuming a form for the evolving spectrum. The wave transport equation is

$$
\frac{\partial F}{\partial t}+\nabla \cdot\left(\mathbf{c}_{\mathbf{g}} F\right)=S_{i n}+S_{n l}+S_{d}
$$

where $F(f, \theta)$ is the wave spectrum as a function of frequency and direction, $\mathbf{c}_{\mathbf{g}}$ is the group velocity, and the terms on the right-hand side represent the source terms: $S_{\text {in }}$ is the energy input due to wind forcing, $S_{n l}$ is the nonlinear energy transfer between groups of resonant waves, and $S_{d}$ is the dissipation of energy due to whitecapping.

[20] In the most recent version of the WAM model (Cycle 4, released in December 1991) the physics included a dynamic coupling between wind and waves [Janssen, 1989, 1991]. Bender and Leslie [1994] and Bender [1996] compared the original Cycle 3 [Snyder et al., 1981; Komen et al., 1984] and Cycle 4 physics to independent buoy data over a 1-month period (July 1992) and concluded that a wave forecasting model for the Australian region should be based on the Cycle 3 physics. Although this led to underprediction of wave heights, it was shown that by upgrading the first-order propagation numerics to third-order and increasing the magnitude of the dissipation term by approximately $40 \%$, a good match with the buoy observations could be achieved. Therefore the present implementation of the WAM model at the Bureau and the version used throughout this work, uses the Cycle 3 physics with increased dissipation and third-order upwinding numerics.
[21] Wave spectra are discretized into 12 directional bins, centered at $15^{\circ}, 45^{\circ}, 75^{\circ}$, etc. This "staggering" of the directional bins is to avoid having spectral energy propagating directly along the axes of the north-south coordinate system [Bidlot et al., 1997]. There are 25 frequency bins ranging from $0.0418 \mathrm{~Hz}$ to $0.4114 \mathrm{~Hz}$. Deep water physics only are used. The propagation and source term time steps are $20 \mathrm{~min}$ and $10 \mathrm{~min}$, respectively. For the global version of the model, the north-south extent of the domain is $78^{\circ} \mathrm{N}$ to $78^{\circ} \mathrm{S}$. Sea-ice is not explicitly included in the model. The wave model is implemented at $0.5^{\circ}$ spatial resolution globally.

[22] Forcing fields for the global wave model are wind velocities at $10 \mathrm{~m}$ above sea level. These are obtained from the Bureau's global atmospheric model: GASP [Seaman et al., 1995]. Surface winds are obtained from the lowest level of GASP via Monin-Obukhov theory with empirical stability functions [Garratt, 1992]. The time period considered in this work is a 4-year period from April 1998 to March 2002. At the beginning of this time period, the $10-\mathrm{m}$ wind fields are instantaneous "snapshots" of the surface and are provided to the wave model at 12-hourly intervals and $2.5^{\circ}$ spatial resolution. These are linearly interpolated in time to 3-hourly intervals, and bilinearly interpolated in space to the resolution of the wave model grid. On 9 December 1998, there was a major upgrade to GASP [NMOC, 1998] in which the spatial resolution was increased from T79 to T239. Note, however, that the spatial resolution of the surface winds used to force the wave model did not change at this time. On 9 April 2001, the frequency and spatial resolution of the wind forcing was increased from 12-hourly to 3-hourly intervals and from $2.5^{\circ}$ to $1^{\circ}$ spatial resolution. In addition, at this time, the wind fields presented to the wave model changed from being instantaneous "snapshots" of the surface fields to being smoothed in time with a 6-hour window. These were similarly interpolated to 1-hourly intervals and the resolution of the wave model grid.

[23] Only wave model hindcasts are considered in this work. These are modeled wave fields which have been forced by surface winds obtained from the data assimilation cycle of the atmospheric model. Ideally, information on short-range forecasts is required. This issue will be discussed in detail in section 6.1. No wave data assimilation is used in the construction of the modeled wave fields. Fields of integrated wave parameters (e.g., SWH) are archived every 6 hours.

\section{ERS-2 Observations}

[24] The satellite altimeter data used are ERS-2 Fast Delivery (FD) observations of SWH. For this work there is no objective quality control applied to the data. Greenslade and Young [2004] performed a validation of the ERS-2 FD data against buoy observations over the time period March 1997 to October 2001. The best-fit between the two data sets was found to be

$$
\begin{array}{ll}
\mathrm{SWH}_{b}=0.1 & \mathrm{SWH}_{F D} \leq 0.72, \\
\mathrm{SWH}_{b}=2.187 \mathrm{SWH}_{F D}-1.582 & 0.72<\mathrm{SWH}_{F D}<1.375, \\
\mathrm{SWH}_{b}=1.153 \mathrm{SWH}_{F D}-0.160 & \mathrm{SWH}_{F D}>1.375,
\end{array}
$$


(a)

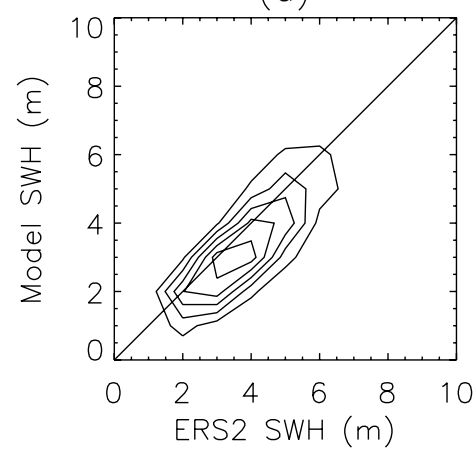

(c)

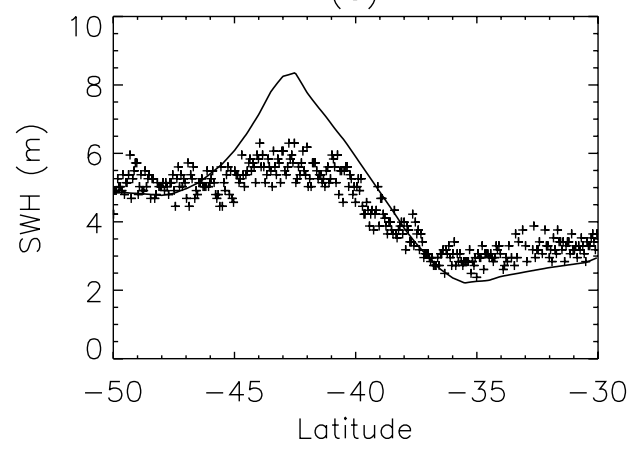

(b)

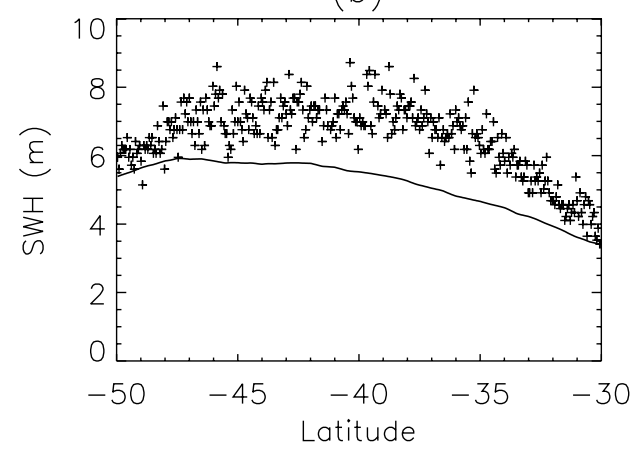

(d)

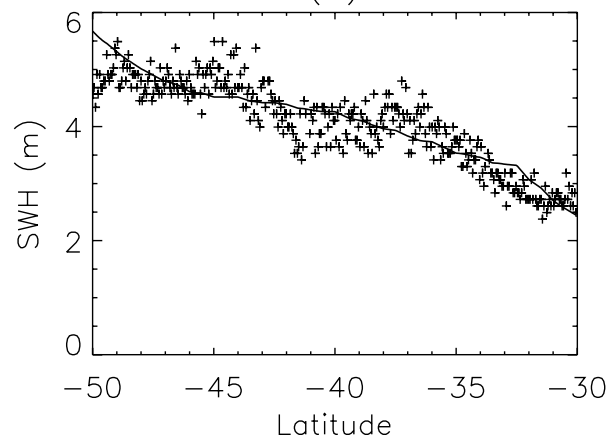

Figure 2. (a) Comparison between model and ERS- $2 \mathrm{SWH}$ for the $20^{\circ}$ box centered at $\left(100^{\circ} \mathrm{E}, 40^{\circ} \mathrm{S}\right)$. Contours are at intervals of 1000 . (b, c, d) Three example overpasses during the 3-month time period October-December 2000. The crosses are the individual ERS-2 observations and the solid line is the bias-corrected model SWH.

where $S W H_{b}$ is the buoy SWH and $S W H_{F D}$ is the ERS-2 FD SWH. Adjusting the ERS-2 FD data according to equation (15) will result in a data set which, when compared to in situ buoy data has an rms error (i.e., goodness-of-fit) of $0.20 \mathrm{~m}$ and negligible overall bias. The ERS-2 errors are therefore assumed to be unbiased. The impact of potential spatial correlations in the ERS-2 errors will be discussed in section 5.2. For the remainder of this work, "ERS-2" data will refer to ERS-2 FD data corrected according to equation (15). Note that the correction described by equation (15) has a discontinuity in its first derivative. This will therefore introduce a discontinuity in the probability distribution of the corrected observations. While this is not expected to be an issue when using the altimeter observations in a data assimilation system, it does mean that care is required when using the corrected observations in validation studies.

[25] GY1 examined the impact of irregular satellite altimeter sampling patterns on estimates of SWH anomaly correlations. A set of climatological anomaly correlations was constructed from modeled SWH fields. These were calculated over the globe at $10^{\circ}$ intervals, within boxes of side length $20^{\circ}$ in latitude and longitude. The modeled wave fields were then sampled at simulated GEOSAT observation locations and the anomaly correlations were recalculated from the simulated altimeter data. A method for which correlations were calculated between observations that were within 2 hours of each other was found to produce slightly more realistic anomaly correlations than a method using only along-track data pairs. This "2-hour" method allowed the inclusion of prior or subsequent same-direction GEOSAT ground-tracks which provided additional information on the full model anomalies in the zonal direction.

[26] However, the GEOSAT satellite and the ERS-2 satellite have different orbit characteristics. The ERS-2 repeat period is 35 days, as opposed to GEOSAT's 17-day repeat period. Thus the ERS-2 ground tracks are angled more meridionally than the GEOSAT ground tracks. This means that within a $20^{\circ}$ box on the ocean surface, the closest overpasses in time for ERS-2 are approximately 10 hours apart, not 2 hours as they are for the GEOSAT altimeter. In order to be able to consider any extra ground tracks within a 2-hour period, it would be necessary to expand the box size to $30^{\circ}$. This increases the computational time substantially, but it was shown in GY1 that the use of the prior and subsequent ground tracks provides only a minor improvement to the results. Therefore, in this work, only along-track data pairs within $20^{\circ}$ boxes are considered.

\section{Method}

[27] For all $20^{\circ}$ boxes over the globe, and for each 3-month period (henceforth called a "season") within the 4 years considered here, the wave model output is first interpolated to the altimeter observation locations. Figure 2a shows a contoured scatter plot of the comparison between modeled SWH and ERS-2 SWH for a $20^{\circ}$ box in the Indian Ocean during October-December 2000. This figure shows that during this time in this region, the model tends to 
Apr - Jun 1998

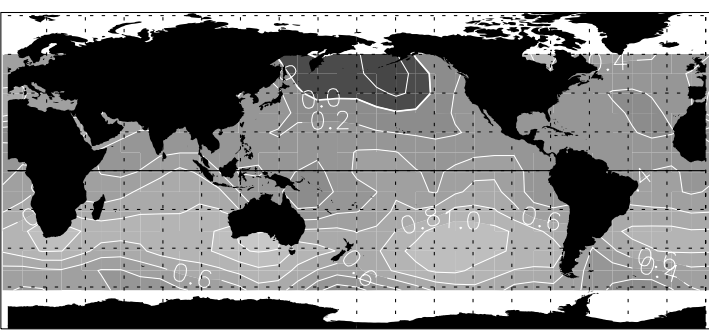

Oct - Dec 1998

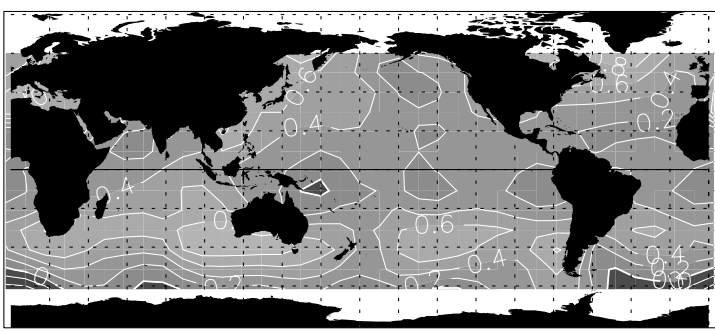

Apr - Jun 1999

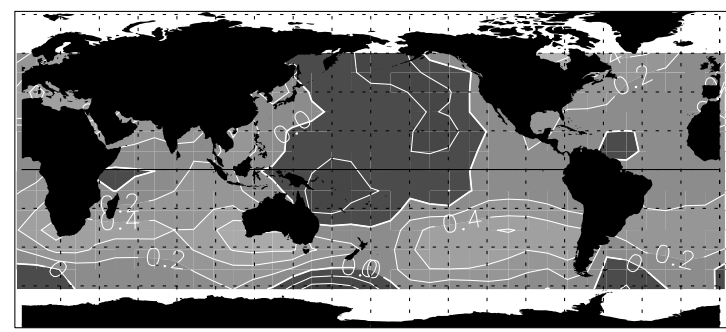

Oct - Dec 1999

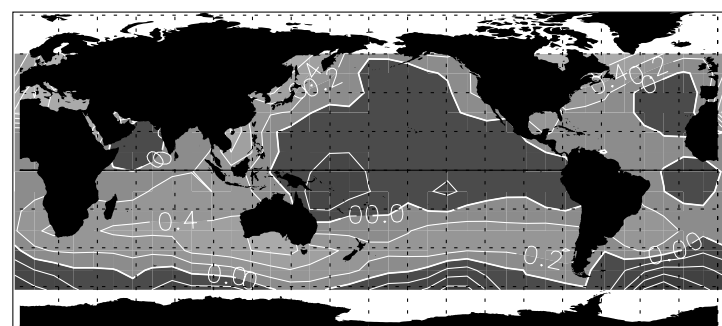

Figure 3. Maps of average modeled SWH minus ERS-2 SWH (m) for each season. Darker regions are where the model SWH $>$ ERS-2 SWH, and lighter regions are where the model SWH $<$ ERS-2 SWH.

underestimate SWH. The average bias (model - ERS-2) is $-0.46 \mathrm{~m}$. Figures $2 \mathrm{~b}, 2 \mathrm{c}$, and $2 \mathrm{~d}$ show the SWH observations from some typical altimeter ground tracks during this period as a function of latitude along with the interpolated model values. Note that the model SWH in Figures $2 b, 2 c$, and $2 d$ has been corrected according to the calculated $-0.46-\mathrm{m}$ bias. The model bias will be discussed further in the next section. These figures show that there is considerable variability in the structure of the model errors. The term "error" is used

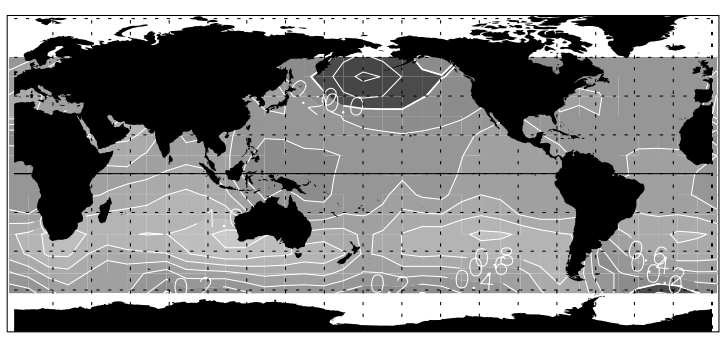

Jan - Mar 1999

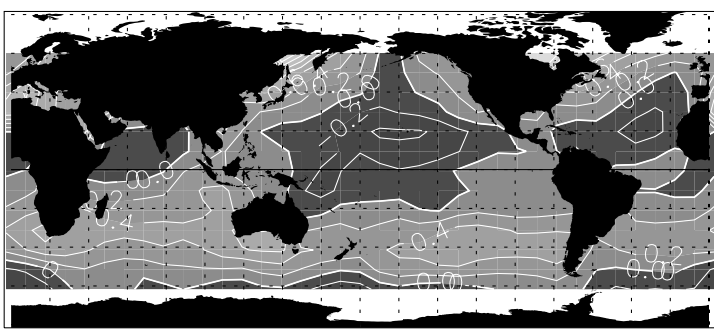

Jul - Sep 1999

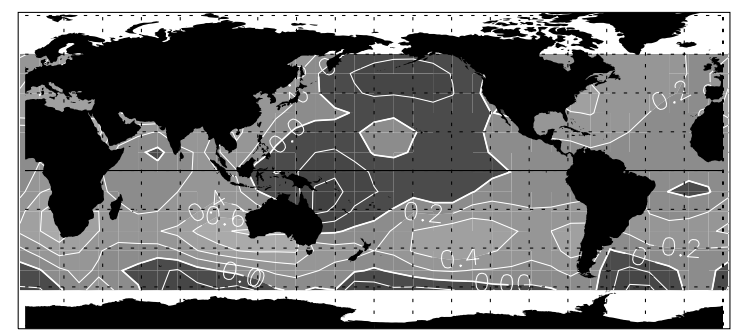

Jan - Mar 2000

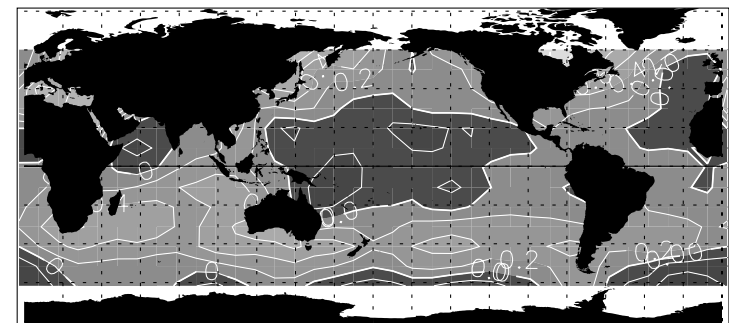

here in its strictest sense, being the difference between the model and the truth. Even though the true SWH is not known, it is assumed that the ERS-2 data are unbiased. In addition, if it is also assumed that subsets of the global ERS-2 SWH data are unbiased, then the true SWH must lie somewhere within the cloud of altimeter observations. In Figure $2 b$, it can be seen that the model underestimates SWH along the entire track, even after taking into account the fact that the model SWH has already been bias-corrected. In 
Apr - Jun 2000

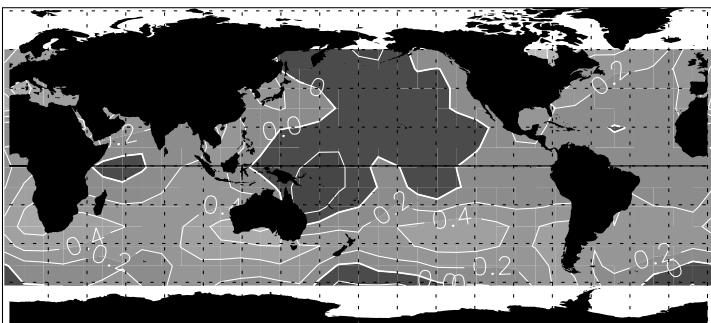

Oct - Dec 2000

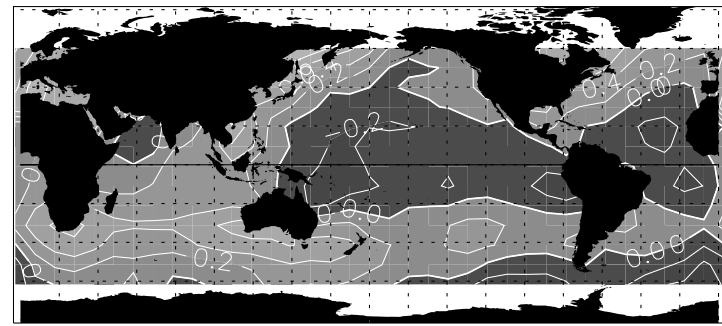

Apr - Jun 2001

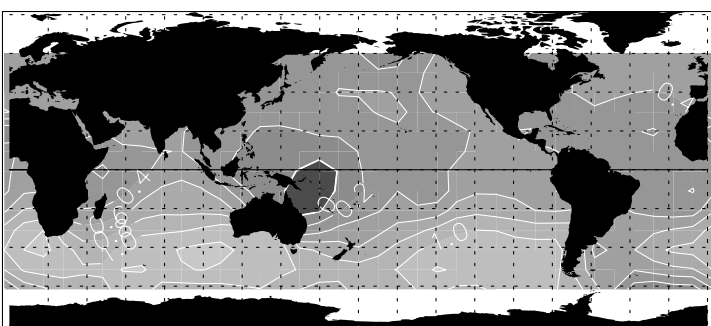

Oct - Dec 2001

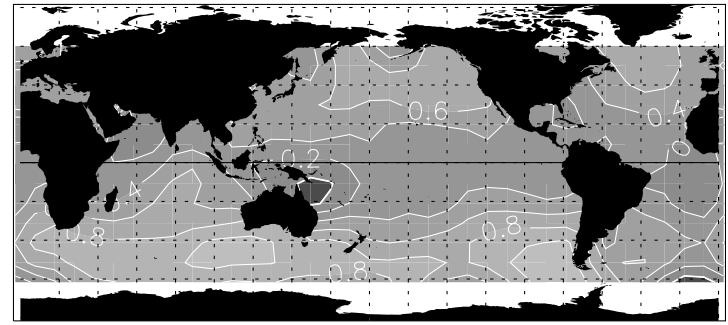

Figure 3.
Jul - Sep 2000

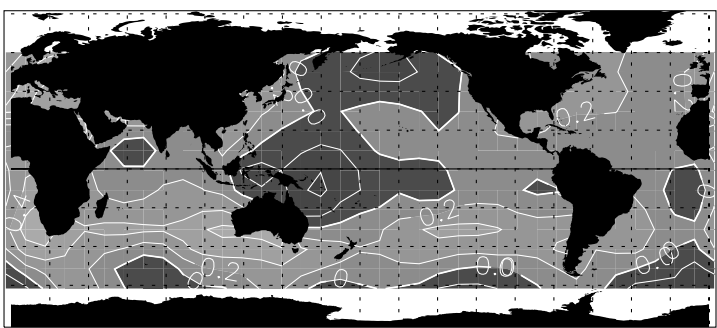

Jan - Mar 2001

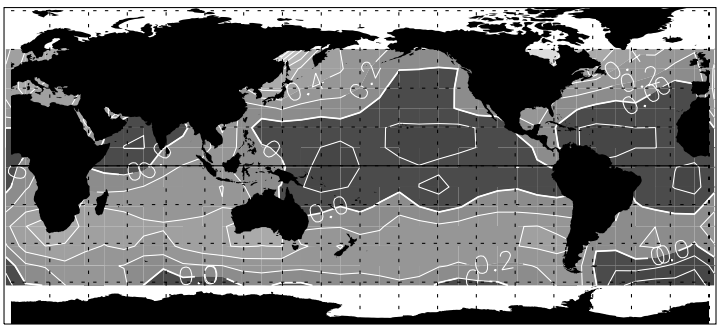

Jul - Sep 2001

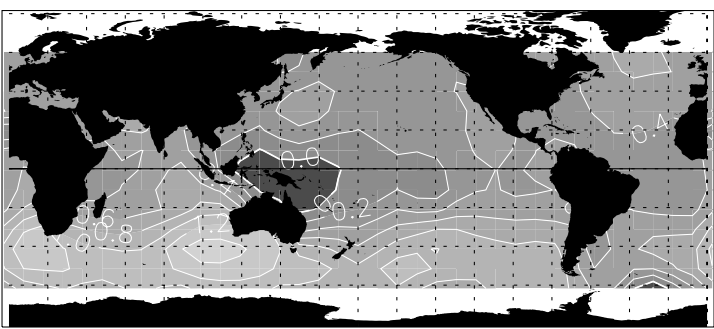

Jan - Mar 2002

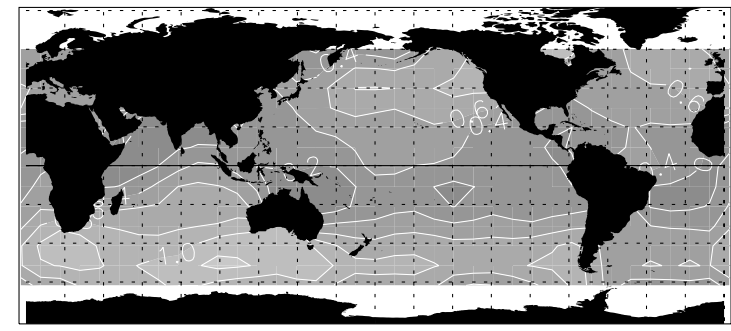

(continued)

At the beginning of this time period, it can be seen that the model generally underestimates SWH over the globe compared to ERS-2. During December 1998, there was a major upgrade to the resolution of the atmospheric model (see section 3). After this upgrade, from 1999 to 2001, a fairly consistent pattern emerges in which the model overestimates SWH in the central Pacific, along the southernmost latitudes and in the mid-Atlantic basins, but underestimates SWH elsewhere. The largest bias is approximately $-0.5 \mathrm{~m}$ 
and occurs in a band at around $30^{\circ} \mathrm{S}$ to $40^{\circ} \mathrm{S}$. An abrupt change to the global distribution of bias occurs during April 2001. At this time, the magnitude of the difference between the wave model SWH and the ERS-2 SWH increases by approximately $0.5 \mathrm{~m}$ over the entire globe. This has the result of reducing the region of positive bias to a small area in the western equatorial Pacific, while over the rest of the globe, the model SWH is severely underestimated. During this latter time period, there are large regions in the Southern Ocean where the model underestimates SWH by over $1 \mathrm{~m}$.

[29] This change in the characteristics of the wave model bias again coincides with a change in the characteristics of the wind forcing fields. During April 2001, the frequency and spatial resolution of the operational global wind forcing was increased and in addition, the winds were smoothed in time (see section 3 ). These changes to the resolution of the wind forcing cause a degradation in the overall model bias. An increased frequency in the wind forcing means that wind variability on shorter time scales is included in the forcing. This should result in increased energy in the waves [Komen et al., 1994], and thus reduce the (negative) bias overall. However, the additional impact of the smoothing in time of the wind fields removes the short timescale variability of the wind fields, and it is this factor which contributes to the increased bias in the SWH fields. It will be seen later, however, that the error variance of the model $\mathrm{SWH}$ is improved substantially with this upgrading of the winds.

[30] The largest biases in SWH during this 4-year time period occur between $30^{\circ} \mathrm{S}$ to $50^{\circ} \mathrm{S}$, and the largest biases within this latitude band generally occur near or just off the southwest coast of Australia. This area of the globe experiences the highest average annual values of SWH [Young, 1999]. This suggests that the bias in modeled SWH may be a constant ratio of the total observed SWH. The mean observed SWH and the mean modeled SWH over the 4 -year period are shown in Figures $4 \mathrm{a}$ and $4 \mathrm{~b}$, respectively. The observed SWH demonstrates that the highest average SWH during this time period actually occurs at around $50^{\circ} \mathrm{S}$, to the southwest of the area of largest bias. Thus the bias is not simply proportional to the SWH. This is confirmed by Figure 4c, which shows the bias in the modeled SWH as a percentage of the observed SWH. The largest value of the bias as a percentage of the observed SWH is still found around the Australian coastline and in the $30^{\circ} \mathrm{S}$ to $40^{\circ} \mathrm{S}$ latitude band. If it is assumed that all the errors in the wave model are due to errors in the wind forcing, then this suggests that the main deficiencies in the atmospheric model are in the westerly winds in the southern latitudes, particularly in the Indian Ocean. This is likely to be associated with the known large-scale, small-amplitude negative bias in sea level pressure of the atmospheric model at high southern latitudes [NMOC, 2003].

[31] The SI theory outlined in section 2 assumes that the background fields are unbiased. Thus it is important to quantify and, if possible, remove any known biases from the modeled wave fields. In an operational environment, this is often not possible. However, it is generally assumed, as it is here, that the biases in the modeled wave fields arise from biases in the surface wind fields. If the systematic errors in the wind fields are known, then they can be removed before
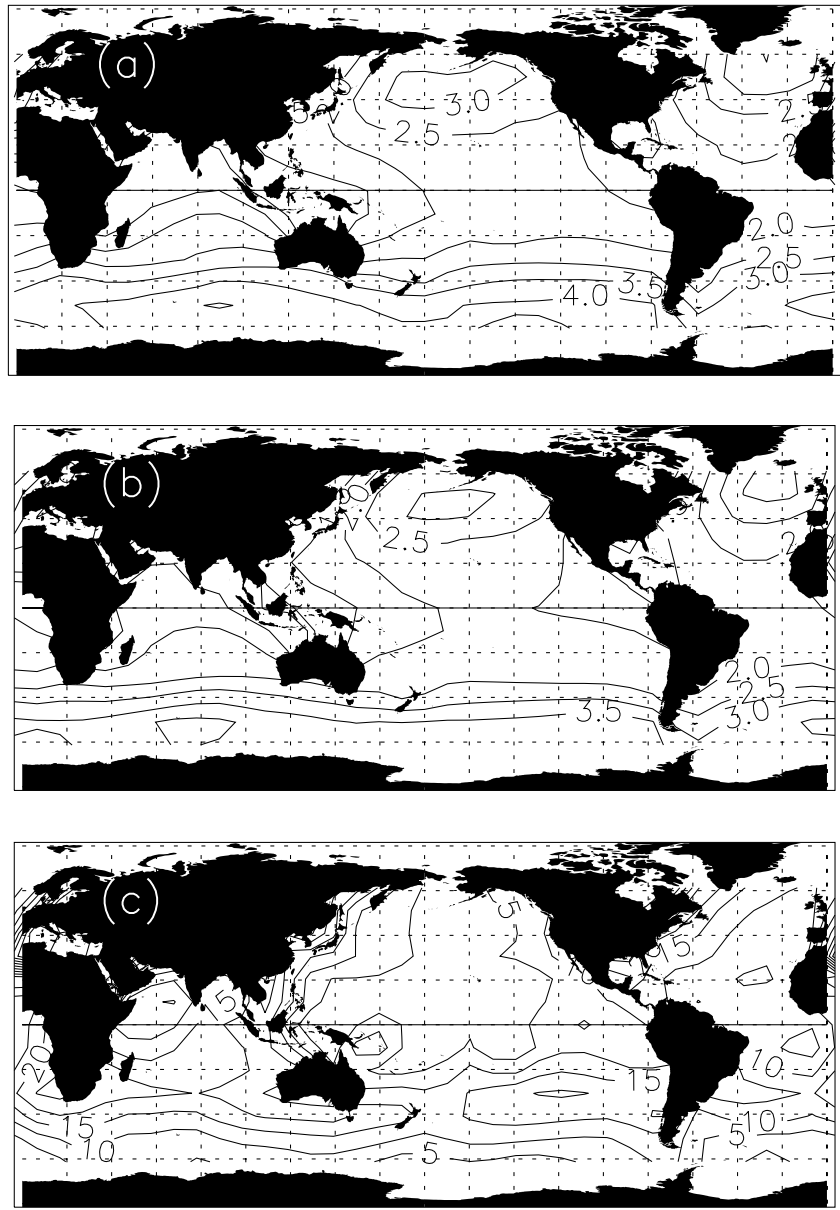

Figure 4. (a) Mean observed (ERS-2) SWH (m) over 4 years. (b) Mean modeled SWH (m) over 4 years. (c) Absolute value of the difference between mean observed and modeled SWH as a percentage of mean observed SWH.

being used to force the wave model [e.g., Tolman, 1998; Kepert et al., 2004, in press].

\subsection{Correlation Computations}

[32] The background error correlation matrix in equation (5) can be expressed as the spatial error correlation between two points, $j$ and $k$, i.e., [Daley, 1991]

$$
R_{j k}=\frac{\overline{\left(O_{j}-B_{j}\right)\left(O_{k}-B_{k}\right)}}{\sqrt{\overline{\left(O_{j}-B_{j}\right)^{2}} \overline{\left(O_{k}-B_{k}\right)^{2}}}}=\rho(r),
$$

where $O_{j}$ is the ERS-2 SWH and $B_{j}$ is the model SWH interpolated to the location of the ERS-2 observation. The overbar represents the time average over the number of time levels that observations occur at both locations $j$ and $k$ during a season (as opposed to the true expected value in equation (5) denoted by the expectation operator: $\langle\ldots\rangle)$. This is therefore the number of altimeter overpasses at a particular location within each $20^{\circ}$ box during each season. Here $\rho(r)$ is the error correlation as a function of great circle distance, $r$. Note that only isotropic correlations are considered; that is, the angle between points $j$ and $k$ is not taken into account. GY1 demonstrated that the satellite 


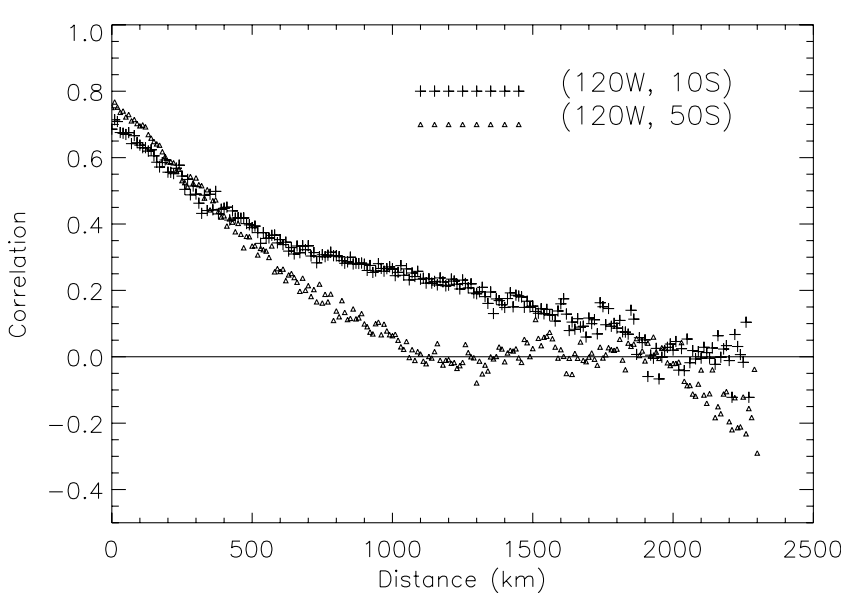

Figure 5. Two model error correlation functions during the time period July-September 2001. These are for $20^{\circ}$ boxes in the Eastern Pacific Ocean centered at $\left(120^{\circ} \mathrm{W}, 10^{\circ} \mathrm{S}\right)$ (crosses) and $\left(120^{\circ} \mathrm{W}, 40^{\circ} \mathrm{S}\right)$ (triangles).

altimeter sampling pattern does not provide enough directional information to enable reliable computation of anisotropic error correlations. However, examination of correlations along ascending and descending ground tracks separately can provide an indication of where anisotropy is important. This will be examined in section 6.2. Before calculation of the correlations, any existing bias must be removed from the observations and the modeled fields. The observations are assumed unbiased, and the seasonal global model bias shown in Figure 3 is removed from the modeled SWH fields for each season.

[33] Two example model error correlations are shown in Figure 5 as a function of distance. Correlations are averaged into $10-\mathrm{km}$ bins, so that, for example, the value of the correlation in the first ( 0 to $10 \mathrm{~km}$ ) bin is the average value of the correlation between all data pairs which are less than $10 \mathrm{~km}$ apart. Since ERS-2 observations are 6 to $7 \mathrm{~km}$ apart, these data pairs will consist of all pairs of adjacent observations. Note that the values of the correlations at zero lag are not equal to 1 . This will be discussed in detail later.

[34] The two correlation functions shown in Figure 5 are clearly different. The rate of decay of the function for the $50^{\circ} \mathrm{S}$ box is more rapid than that for the $10^{\circ} \mathrm{S}$ box, and at a spatial lag of $1000 \mathrm{~km}$, the value of the correlation for the $10^{\circ} \mathrm{S}$ box is around 0.3 , while the value of the correlation in the more southerly box has reached zero. This indicates that the spatial scale of the model errors is larger in the $10^{\circ} \mathrm{S}$ region than in the $50^{\circ} \mathrm{S}$ region.

[35] At long spatial lags, the correlations tend to be noisy and do not necessarily equal zero. This is a reflection of the fact that there are fewer data pairs with large distances between them. The fact that the correlations do not always equal zero at long lags can have an adverse effect on the process of finding the best-fit analytic representation for the functions. This could be overcome by several methods. One option could be to weight the value in each bin according to the variance within the bin or according to its spatial lag. Another option could be to truncate the correlation function at a particular lag before applying the curve-fitting procedure. The disadvantage of this method is that in this case, it is not obvious at what distance the function should be truncated. Another way to address this issue could be to expand the box size considered. However, the end points would still be noisy, they would just be at longer distances. Hence it is not clear that this would justify the increase in computational time. A fourth method could be to consider a time period longer than 3 months so that more satellite overpasses are included in the correlation computations. The disadvantage of this method is that it makes it difficult to examine seasonal variability.

[36] In this work, the total time period that is being examined is 4 years. It is possible to simulate a time period longer than 3 months by averaging together correlations from different seasons. It was found that four seasons were sufficient to ensure that the correlations approach zero at long lags. The seasons that are averaged together may be sequential or they may be the same season in different years. There is the issue, however, that averaging together correlations from different seasons is not exactly the same as considering a correlation over a longer time period. However, under stationary conditions, the variance $\overline{\left(O_{j}-B_{j}\right)^{2}}$ will remain constant (where the overbar is the average over the number of times that observations occur at location $j$ ). Examination of equation (16) shows that with this assumption, correlations from different time periods may be averaged together. It will be seen later however that the model error variance $\overline{\left(B_{j}-T_{j}\right)^{2}}$ is affected when the characteristics of the wind forcing changed during April 2001, and so this assumption is not strictly valid. In addition, both the model and the observational error variances have some seasonal variability. This should be borne in mind during the presentation of the results of the next section.

\subsection{Error Variances}

[37] As mentioned previously, an important feature of the isotropic correlation functions shown in Figure 5 is that the value of the correlation at zero lag is not equal to 1 , and by definition $R_{k k}=1$. Note that this is not really zero lag because there are no data pairs that are $0 \mathrm{~km}$ apart, but it can easily be seen in Figure 5 that the zero-intercept of a fitted curve should be very close to the value in the 0 to $10 \mathrm{~km}$ bin.

[38] Following Daley [1991], an analytic correlation function, $\rho(r)$, is defined to be the best-fit curve to any particular correlation function. Then

$$
R_{0}=\lim _{r \rightarrow 0} \rho(r)
$$

The ERS-2 altimeter SWH errors are assumed to be spatially uncorrelated, so their only contribution to $\rho(r)$ will be at $r=0$. On the other hand, the model errors are spatially correlated. $R_{0}$ is a measure of the spatially correlated component of the total error and is given by

$$
R_{0}=\frac{E_{B}^{2}}{E_{B}^{2}+E_{O}^{2}},
$$

where the model error variance $E_{B}^{2}$ and the observation error variance $E_{O}^{2}$ are given by

$$
E_{B}^{2}=\frac{1}{K} \sum_{k=1}^{K} \overline{\left(B_{k}-T_{k}\right)^{2}} \quad \text { and } \quad E_{O}^{2}=\frac{1}{K} \sum_{k=1}^{K} \overline{\left(O_{k}-T_{k}\right)^{2}}
$$


(a)

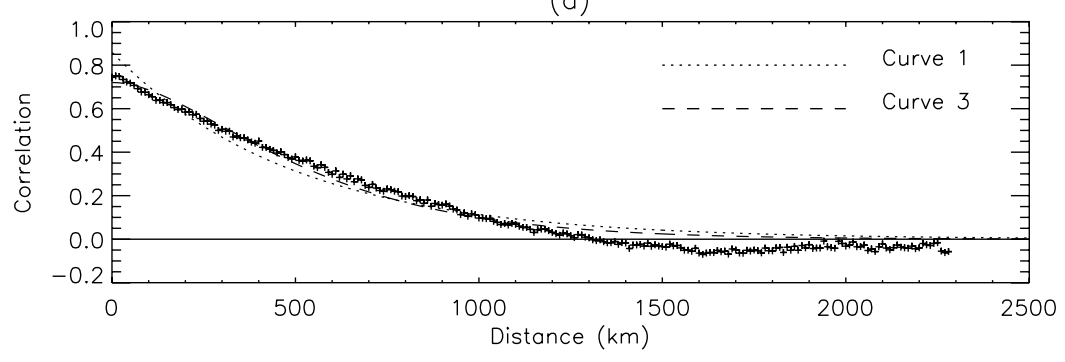

(b)

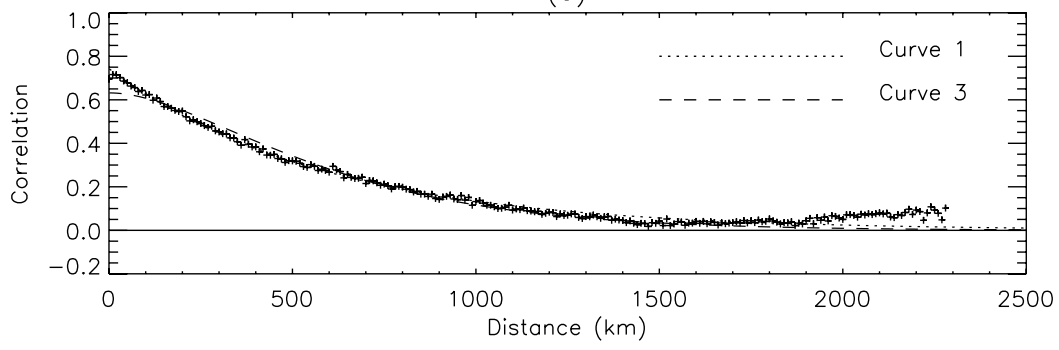

Figure 6. Model error correlation functions (crosses) averaged over 4 years for $20^{\circ}$ boxes in the Indian Ocean centered at (a) $\left(100^{\circ} \mathrm{E}, 30^{\circ} \mathrm{S}\right)$ and $(\mathrm{b})\left(80^{\circ} \mathrm{E}, 20^{\circ} \mathrm{S}\right)$. The dotted line in each panel is the best-fit curve 1 , and the dashed line is the best-fit curve 3 .

where $K$ is the number of locations at which multiple observations occur. The spatially uncorrelated part of the error $\left(E_{O}^{2}\right)$ is made up of the observation error plus the error in spatial scales which are too small to be resolved by the altimeter.

[39] It is possible to find solutions for $E_{B}^{2}$ and $E_{O}^{2}$. If the model errors and observation errors are not correlated with each other, then the mean variance

$$
\begin{aligned}
\frac{1}{K} \sum_{k=1}^{K} \overline{\left(O_{k}-B_{k}\right)^{2}} & =\frac{1}{K} \sum_{k=1}^{K} \overline{\left(O_{k}-T_{k}\right)^{2}}+\frac{1}{K} \sum_{k=1}^{K} \overline{\left(B_{k}-T_{k}\right)^{2}} \\
& =E_{B}^{2}+E_{O}^{2} .
\end{aligned}
$$

Since $R_{0}$ can be determined by finding appropriate analytic curves to describe the correlation functions, and $\frac{1}{K} \sum_{k=1}^{K} \overline{\left(O_{k}-B_{k}\right)^{2}}$ can easily be computed for each season, then equations (18) and (20) can be solved for $E_{B}^{2}$ and $E_{O}^{2}$.

[40] As mentioned previously, recent work has shown that there may be some spatial correlation in the observations on scales of approximately $70 \mathrm{~km}$ [Janssen et al., 2003]. This has not been taken into account in this work. Spatial correlations in the observations would result in a lower value of $\rho(r)$ on these scales, and therefore a lower value of $R_{0}$. This in turn would result in a larger value of the calculated observational error variance. In other words, it is possible that by ignoring the spatial correlations in the observations, the observational error has been underestimated. It will be seen later that the spatial scales of the model error are considerably larger than the spatial scales of the observational error. This means that the neglect of any spatial correlations in the observational error will not have a significant impact on the determination of the spatial scales of the model error.

\subsection{Fitting to Analytic Functions}

[41] The calculated anomaly correlations $\rho(r)$ were fitted to analytic functions. The specific functions tested were selected in order to include all the functions that are currently used operationally (see section 2). In addition, the functions suggested by Julian and Thiébaux [1975] were tested. All the tested functions were positive definite, a requirement of the SI procedure. The "best" six curves are listed below.

Curve 1

$$
\rho(r)=a_{2} \exp \left[-\frac{1}{2}\left(\frac{r}{a_{1}}\right)\right]
$$

Curve 2

$$
\rho(r)=a_{2} \exp \left[-\frac{1}{2}\left(\frac{r}{a_{1}}\right)^{2}\right]
$$

Curve 3

$$
\rho(r)=a_{2}\left(1+\frac{r}{a_{1}}\right) \exp \left[-\frac{r}{a_{1}}\right],
$$

Curve 4

Curve 5

$$
\rho(r)=a_{2}\left(1+\frac{r}{a_{1}}\right) \exp \left[-\frac{1}{2}\left(\frac{r}{a_{1}}\right)\right]
$$

$$
\rho(r)=a_{2}\left(1+\left(\frac{r}{a_{1}}\right)^{2}\right)^{-1}
$$

Curve 6

$$
\rho(r)=a_{2}\left(1+\left(\frac{r}{a_{1}}\right)^{2}\right)^{-\frac{1}{2}} .
$$

To find the most appropriate functional form, the minimization was performed on the correlations that are obtained when all 16 seasons are averaged together. For each Curve 1 to 6 in equation (21), and for each $20^{\circ}$ box, the parameters $a_{i}$ that resulted in the lowest mean-square-error (mse) were determined. Figure 6 shows examples of a correlation function for two locations and the best-fit for Curves 1 and 3 . It can be seen from this figure that there is not an obvious 


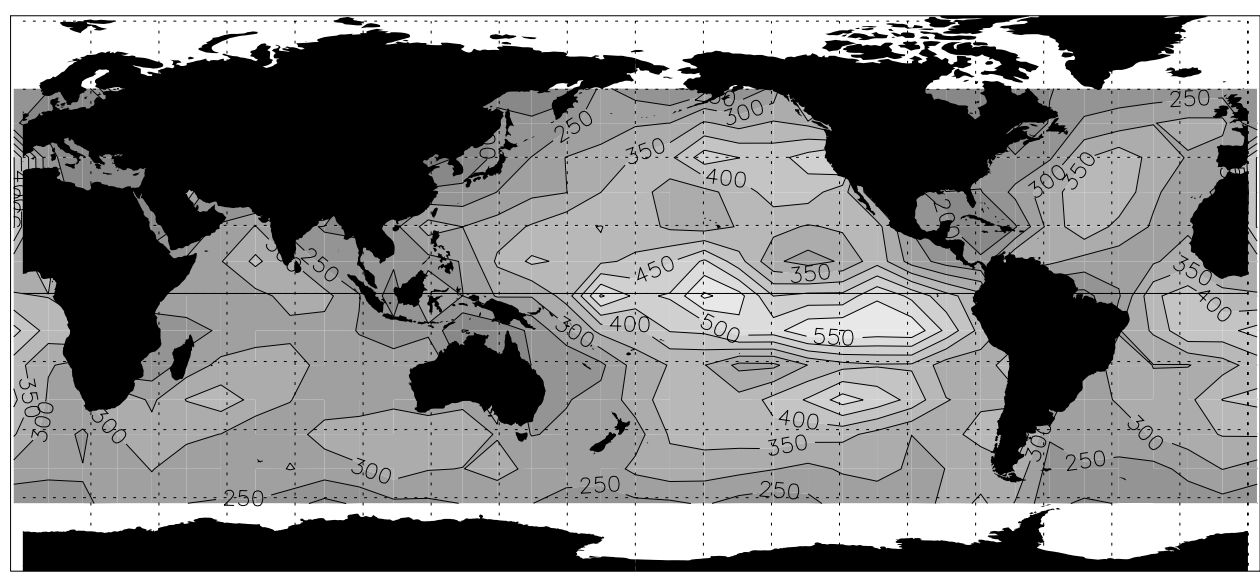

Figure 7. Average model error correlation length scale over the 4-year period April 1998 to March 2002 (uncorrected).

choice between either curve. The values of the mse for each curve for $\left(100^{\circ} \mathrm{E}, 30^{\circ} \mathrm{S}\right)$ are Curve 1: 0.003, Curve 3: 0.0016 and for $\left(80^{\circ} \mathrm{E}, 20^{\circ} \mathrm{S}\right)$ are Curve 1: 0.0006, Curve 3: 0.0011 . Therefore, overall, Curve 3 represents the top correlation better, but Curve 1 fits the bottom correlation better. Neither curve matches the zero-intercept well for both locations and this is an issue when determining $E_{O}^{2}$ and $E_{B}^{2}$. However, there are ways to avoid this problem, and this is discussed in the next section.

[42] The average mse over all $20^{\circ}$ boxes (excluding boxes with significant amounts of land) for each curve was calculated. Curve 1 and Curve 3 resulted in the lowest mse and performed equally well. It is possible that one particular curve may be appropriate for some specific geographic regions, while the other is suitable in other areas. This possibility was examined; however, no distinct regions were found where one curve was seen to be more appropriate. Another avenue that was examined was to determine which curve overall had the smallest error at $r=0$, where the error is defined as the difference between the fitted value of $\rho(0)$ and the value of the correlation in the 0 to $10 \mathrm{~km}$ bin. Again, the two curves performed equally well. Here, Curve 3 , the auto-regressive curve, is chosen as this will be consistent with the results of GY1. The model error correlation length scale calculated from ERS-2 altimeter data, $L$, is therefore defined to be $a_{1}$ in Curve 3. Figure 7 shows a contour map of $L$ for the case with all sixteen seasons averaged together. Before discussing the global distribution of model error correlation scales shown in Figure 7, it is necessary to make some corrections to the length scales. These corrections are necessary because of the irregular sampling pattern of the satellite altimeter.

\subsection{Adjustments to the ERS-2 Length Scales}

[43] In GY1, it was shown that due to the sparse sampling pattern of satellite altimeters, anomaly correlation length scales obtained using simulated altimeter data were generally underestimated, particularly at low latitudes. In this section, an appropriate correction to apply to the background error correlation length scales obtained from ERS-2 data is determined. A set of model anomaly correlations for two 3-month time periods is constructed. These are obtained by calculating equation (16) with $O_{i}$ defined as the individual 6-hourly modeled SWH and $B_{i}$ defined as the 3-month mean modeled SWH, i.e.,

$$
B_{i}=\overline{O_{i}}=\frac{1}{N_{t}} \sum_{t=1}^{N_{t}} O_{i}(t)
$$

where $N_{t}$ is the number of 6-hourly model fields within the 3 -month time period. The procedure is identical to that followed in GY1, and further details of the calculations can be found there. The resulting global isotropic anomaly correlation length scales for the two 3-month periods are shown in Figure 8.

[44] The modeled SWH fields were then sampled at simulated ERS-2 observation locations and the model anomalies were reconstructed from this simulated ERS-2 data. The resulting global distributions of $L$ from simulated ERS-2 data $\left(L_{\text {ers }}\right)$ for the two 3-month periods are shown in Figure 9. The length scales from the simulated altimeter data are generally shorter. However, some of the major features of the full model anomaly correlations are evident. For example, during July-September 1998, long length scales appear in the eastern Pacific and along the southernmost latitude band and there is an indication of longer length scales in the northern Indian Ocean (associated with the monsoon). For the second time period, ERS-2 has detected the longer scales in the North Atlantic and in the Eastern Pacific.

[45] The difference, $L_{\text {diff }}$, between the distribution of $L_{\text {ers }}$ in Figure 9 and the "true" $L$ in Figure 8 is shown in Figure 10 for each time period. $L_{\text {diff }}$ is defined here as

$$
L_{\text {diff }}=100 \times \frac{L-L_{e r s}}{L} .
$$

It can be seen that the altimeter does indeed underestimate $L$ over most of the globe. The areas where $L$ is underestimated by the largest amount are at low latitudes. It is not clear why this should be so. One possible reason could be that the characteristics of the altimeter sampling pattern change with latitude, and so this results in a different impact. Another possibility is that the impact of the sampling pattern is dependent upon the "true" length scale and this in turn varies considerably with latitude. 

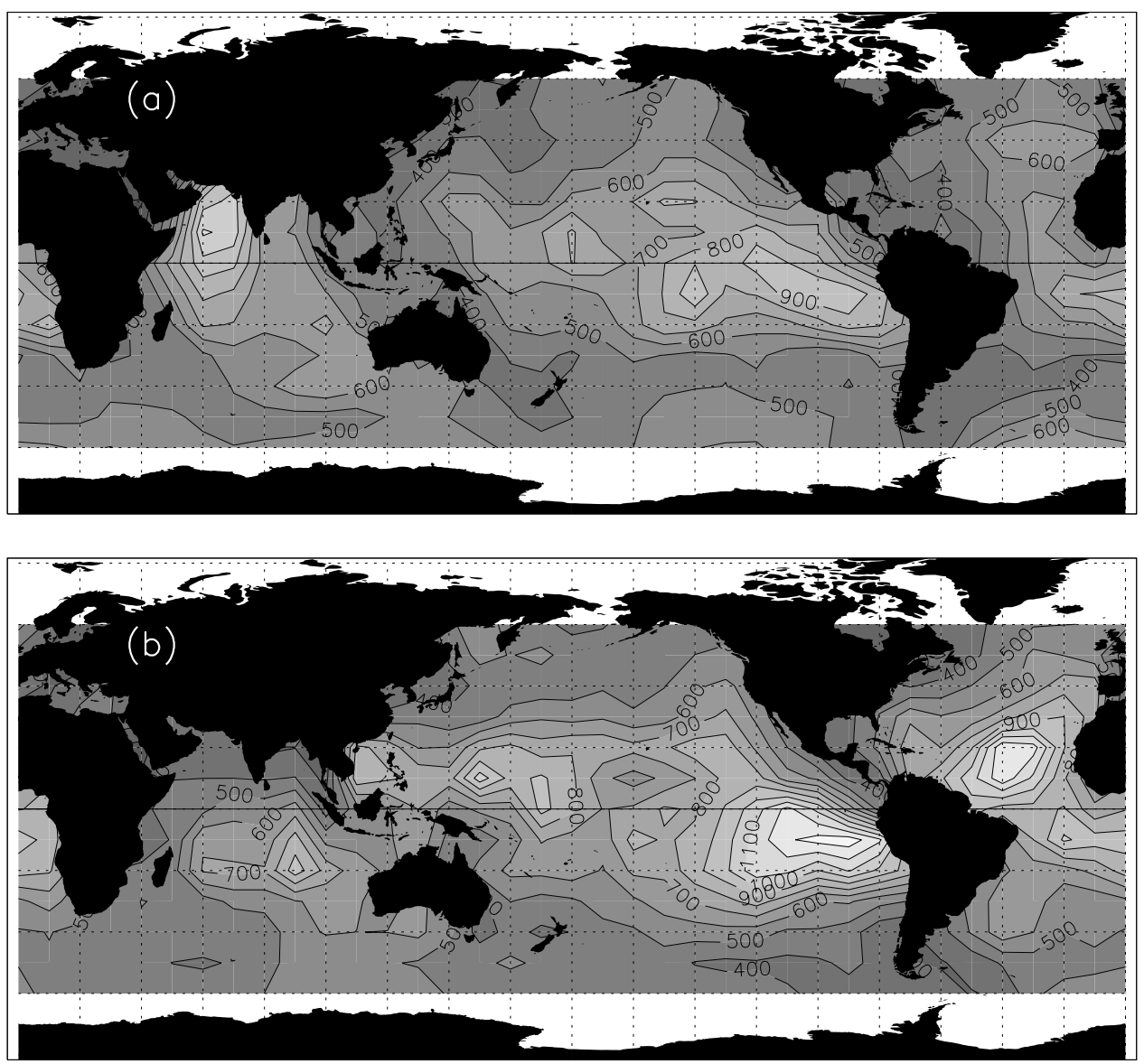

Figure 8. (a) Isotropic anomaly correlation length scale $(\mathrm{km})$ for modeled SWH over the globe for $20^{\circ}$ boxes at $10^{\circ}$ intervals for the time period July to September 1998. (b) Isotropic anomaly correlation length scale $(\mathrm{km})$ for modeled SWH over the globe for $20^{\circ}$ boxes at $10^{\circ}$ intervals for the time period January to March 1999.

[46] These two relationships, i.e., the relationship between $L_{d i f f}$ and $L_{e r s}$ and the relationship between $L_{\text {diff }}$ and latitude are examined here. These are shown as scatterplots in Figure 11. It is assumed that the latitudinal effect is due to the changing pattern of the altimeter sampling with latitude. This is the same in the Northern and Southern Hemispheres and so in this section, no distinction is made between northern and southern latitudes. It can be seen that there are quite strong relationships between the amount by which the ERS-2 sampling pattern underestimates $L$ and both of these factors. The value of the correlation between $L_{\text {diff }}$ and $L_{\text {ers }}$ is -0.52 and between $L_{\text {diff }}$ and latitude is -0.40 .

[47] The inverse correlation between $L_{d i f f}$ and $L_{e r s}$ indicates that the larger the estimated $L_{\text {ers }}$, the smaller the amount by which the true $L$ is underestimated. The underestimation possibly even becomes negative, indicating that in general, when the estimated $L_{\text {ers }}$ is very large, then the true length scale has been overestimated.

[48] There is also a negative correlation between $L_{\text {diff }}$ and latitude, indicating that the altimeter performs better at higher latitudes than at lower latitudes. This is likely to be due to the fact that at higher latitudes, the altimeter sampling pattern covers a wider range of angles and so there is more opportunity to detect both longer and shorter length scales.
At low latitudes, the ground tracks are constrained within a small range of angles, and so the estimate of $L$ is dependent on the anisotropy of the true correlation function and how the anisotropy is positioned relative to the satellite ground tracks.

[49] Figure 12a shows a scatterplot of the comparison between $L_{e r s}$ and $L$. The statistics listed in the figure show how the raw $L_{e r s}$ compares to $L$. These indicate that on average, $L_{e r s}$ is $110.2 \mathrm{~km}$ shorter than $L$ with a standard deviation of $164.3 \mathrm{~km}$. An appropriate adjustment to apply to $L_{\text {ers }}$ is sought based on both latitude $(\phi)$ and $L_{\text {ers }}$. The following simple relationship is assumed:

$$
L=a_{0}+a_{1} \phi+a_{2} L_{e r s} .
$$

Linear regression analysis yields

$$
L_{a d j}=434.4-4.59 \phi+0.607 L_{\text {ers }} .
$$

These adjusted length scales are compared to $L$ in Figure 12b. The comparison statistics in this figure show that with this simple adjustment, the bias is eliminated and the standard deviation is reduced to $129.5 \mathrm{~km}$. Note, however, that in general, high values of $L$ tend to be underestimated by the altimeter and low values of $L$ tend to 

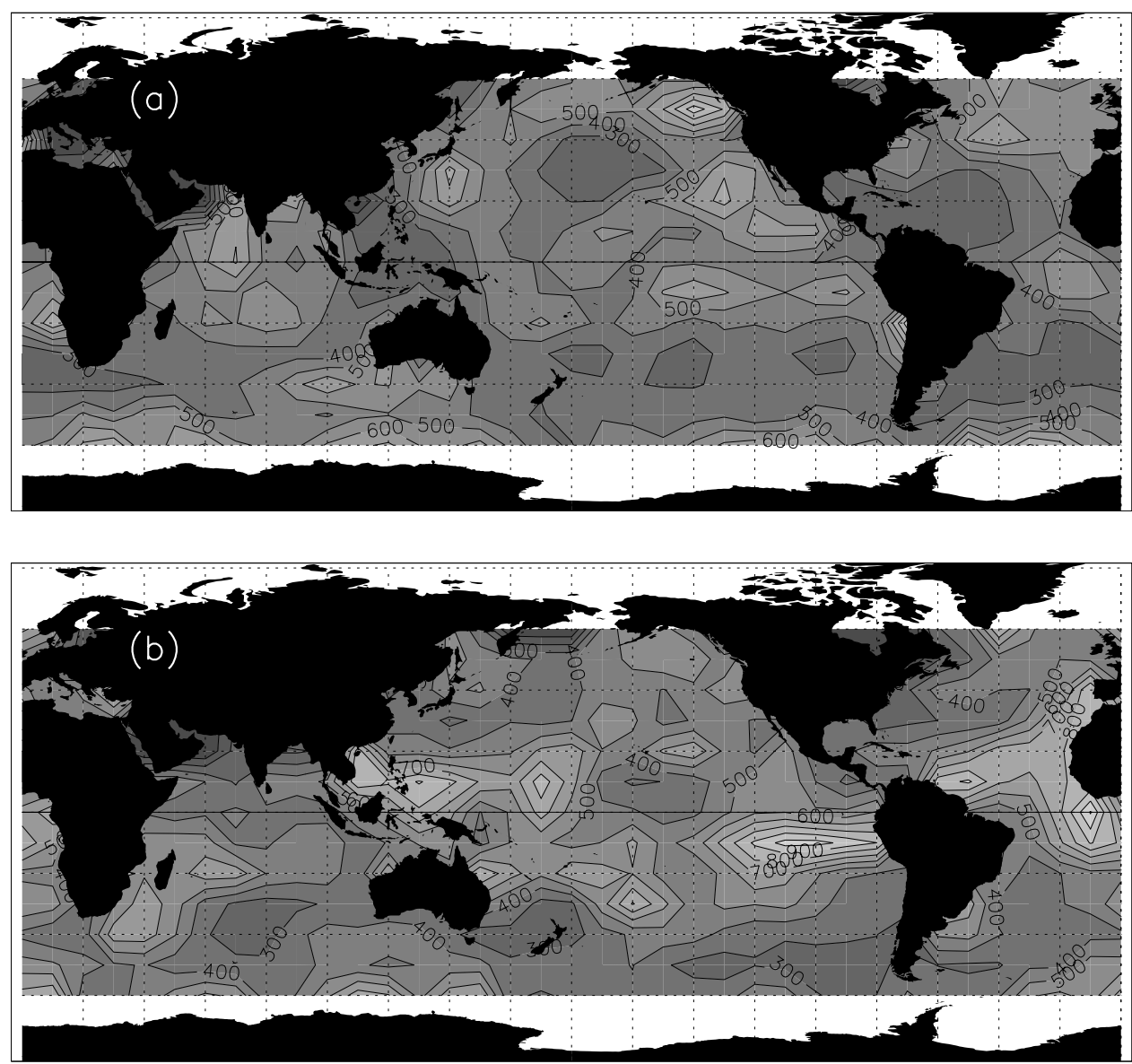

Figure 9. (a) Isotropic length scale of the model anomaly using simulated ERS-2 data for July to September 1998. (b) Isotropic length scale of the model anomaly using simulated ERS-2 data for January to March 1999.

be overestimated. This suggests that the relationship between $L$ and $L_{e r s}$ is more complex than the linear relationship assumed in equation (24). It may be possible to reduce the standard deviation further by exploring nonlinear dependencies. This is left for further work. Applying the correction in equation (24) to $L_{\text {ers }}$ results in the global distributions of the isotropic anomaly length scale shown in Figure 13. These are considerably closer to the "true" distributions of the anomaly correlation length scale than those shown in Figure 9.

\section{Results}

\subsection{Isotropic Correlations}

[50] Applying the correction found in the previous section to the length scales of the model error shown in Figure 7 provides the final result shown in Figure 14. It can be seen from this figure that the scale of the model errors does vary quite significantly over the globe, with a strong latitudinal dependence, ranging from around $300 \mathrm{~km}$ at the highest latitudes to over $600 \mathrm{~km}$ at the equator in the Indian and Atlantic Oceans and $750 \mathrm{~km}$ in the Pacific.

[51] A general explanation of these results could be as follows. Wind waves are generated in the high latitudes, where the winds are generally stronger. The spatial extent of the wave systems is relatively small when they are generated. As they propagate toward low latitudes and become swell, they disperse and thus their spatial scale increases. If these swell systems are incorrectly modeled in the generation region then the spatial scale of the errors will increase toward low latitudes. Examples of incorrect generation of swell could be that the wind speed is biased low and thus the amplitude of the swell is underestimated, or the direction of the winds is incorrect and thus the swell propagates in the wrong direction. There could also be errors in the propagation of swell within the wave model, even if the forcing winds are accurate.

[52] It should be noted here that although none of the studies described in section 2 specifically incorporated geographically varying background errors explicitly, some of the studies [e.g., Lionello et al., 1992] defined the length scale of the background error correlations as a function of degrees of latitude and longitude, or model gridpoints, rather than kilometers. This means that the model errors are defined to have a larger zonal spatial scale at low latitudes than at high latitudes, resulting in both anisotropy and inhomogeneity. Interestingly, the results presented here show that a background error correlation length scale defined in degrees is likely to represent the global distribution of model errors more accurately than one for which $L$ is defined in kilometers. 

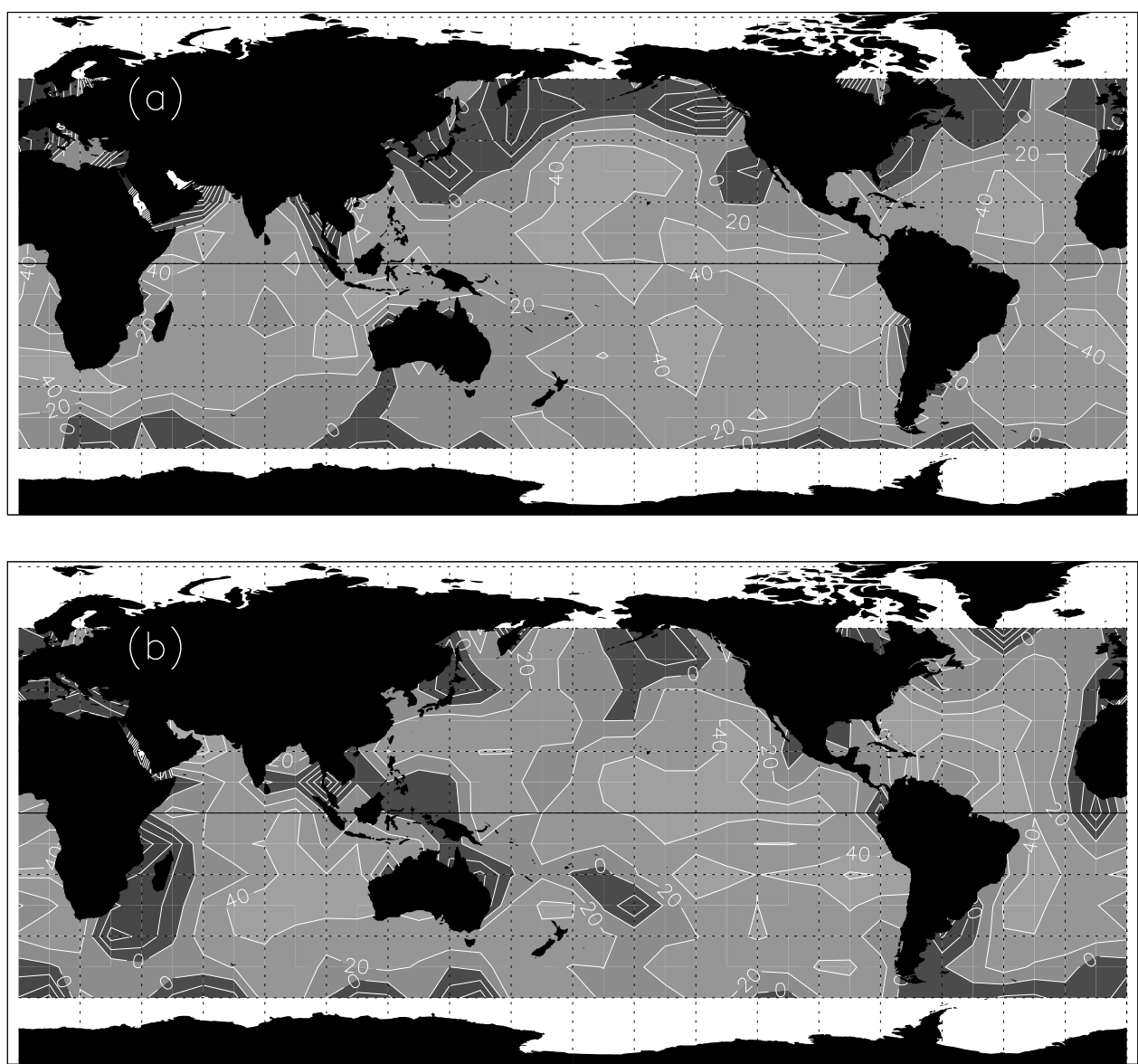

Figure 10. (a) $L_{\text {diff }}$ for simulated ERS-2 data for July to September 1998. (b) $L_{\text {diff }}$ for simulated ERS-2 data for January to March 1999.
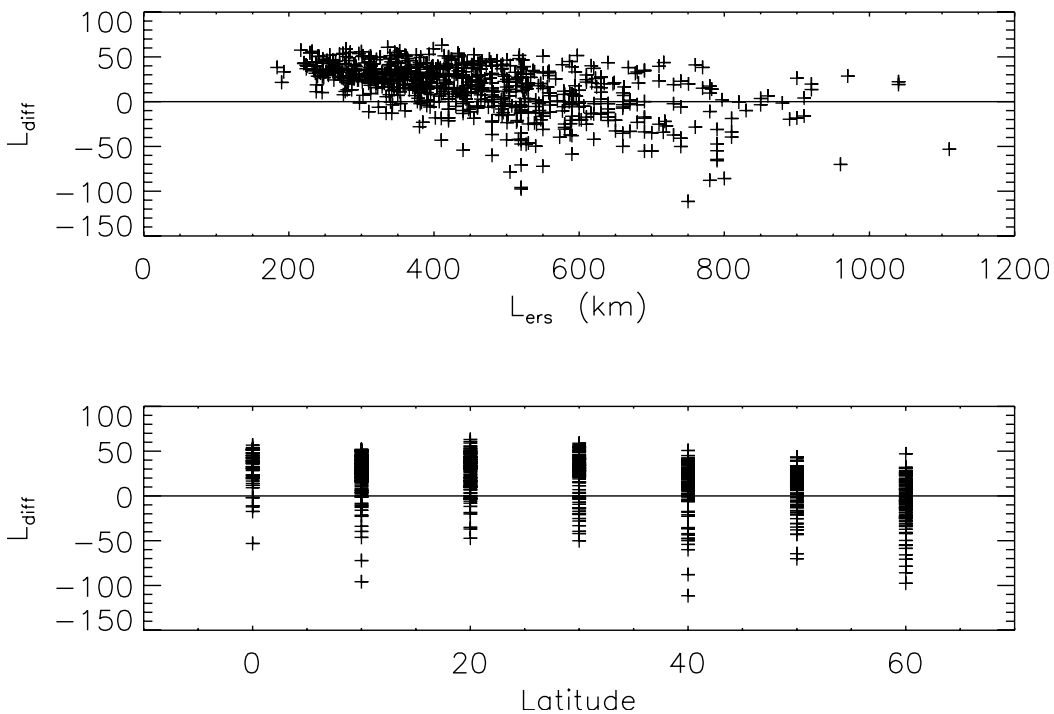

Figure 11. $L_{\text {diff }}$ as a function of latitude and $L_{\text {ers }}$. 
(a)

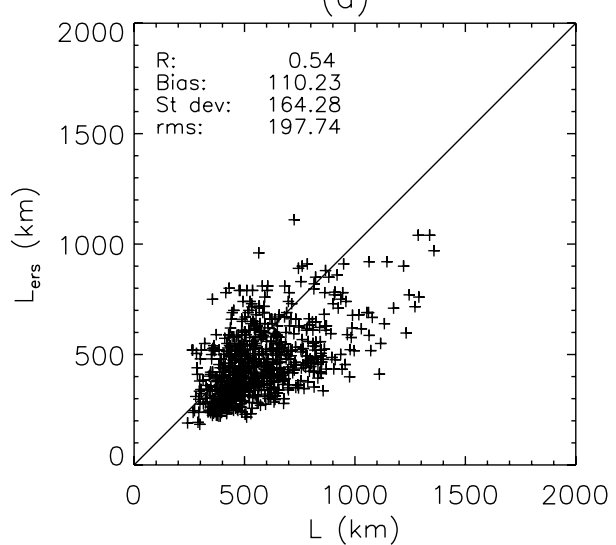

(b)

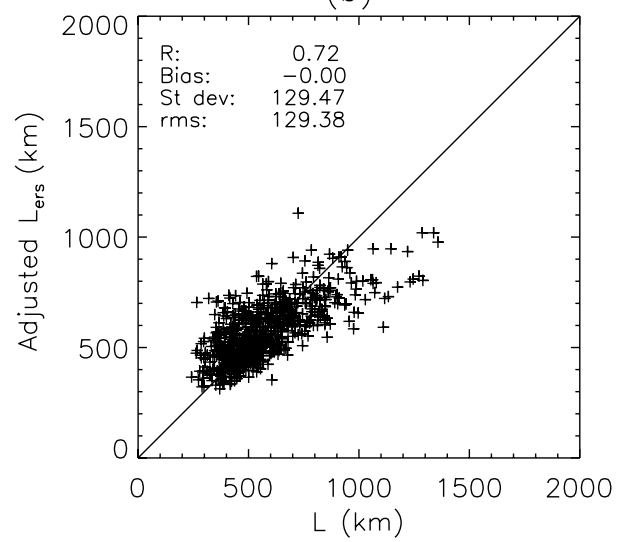

Figure 12. (a) Scatter plot of $L_{\text {ers }}$ versus $L$, (b) Scatter plot of adjusted $L_{e r s}$ versus $L$.
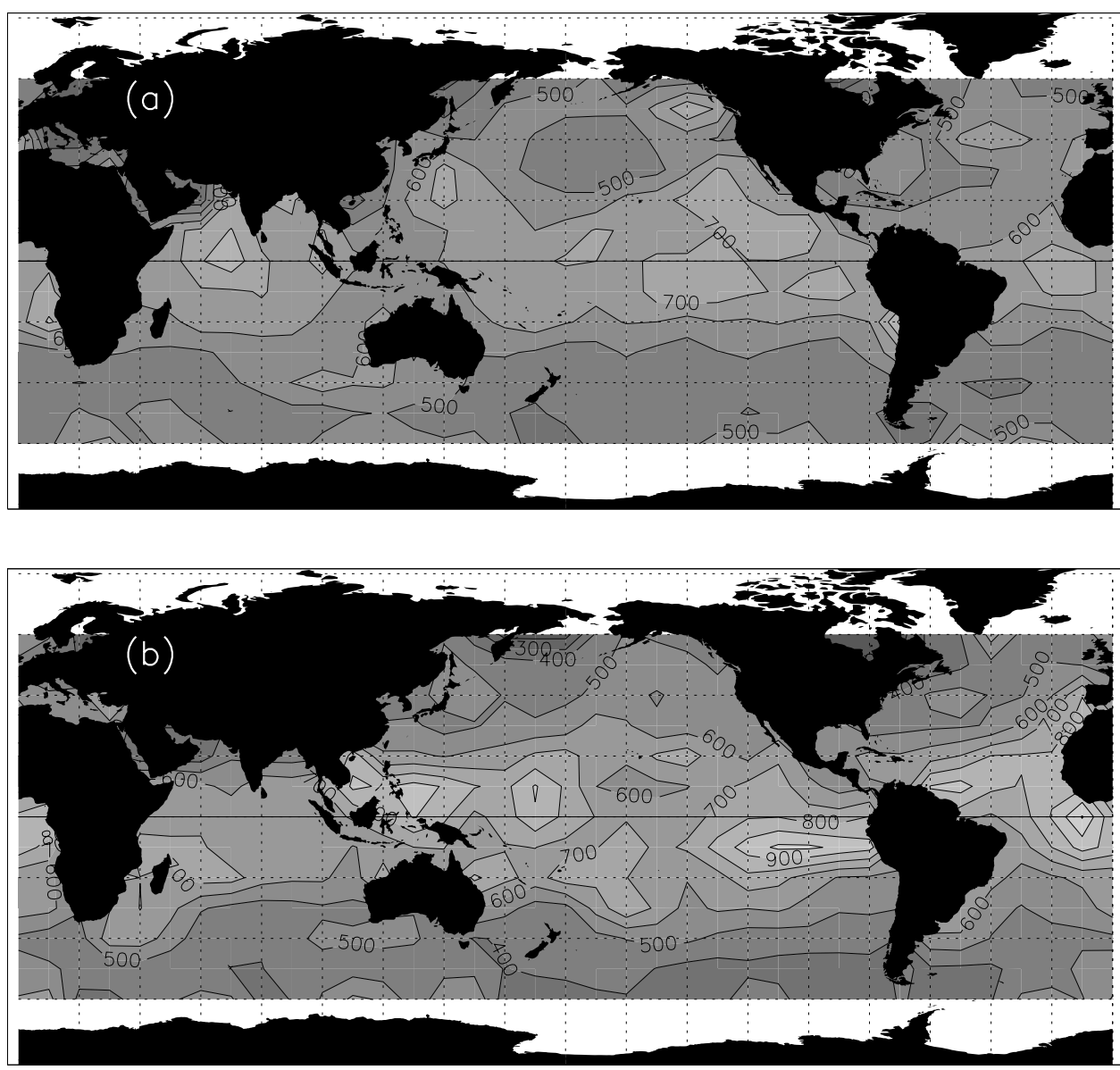

Figure 13. (a) Isotropic correlation length scale over the globe from simulated ERS-2 altimeter data adjusted according to equation (25) for July to September 1998. (b) Isotropic correlation length scale over the globe from simulated ERS-2 altimeter data adjusted according to equation (25) for January to March 1999 . 


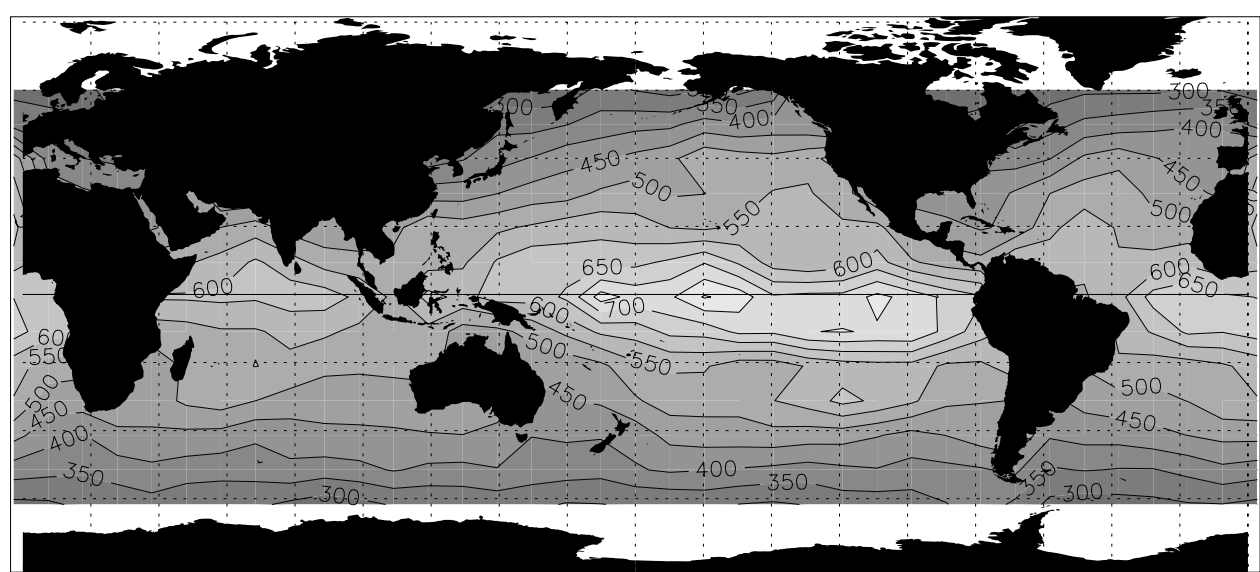

Figure 14. Isotropic model error correlation length scale over the globe from ERS-2 altimeter data adjusted according to equation (25).

[53] It is interesting to compare these results with the background error correlation matrix that is currently used operationally at the Bureau. The current Bureau implementation is [Greenslade, 2001]

$$
P_{k j}=\exp \left[-\frac{1}{2}\left(\frac{\left|x_{k}-x_{j}\right|}{L}\right)^{2}\right]
$$

with $L=300 \mathrm{~km}$. This value was chosen based on the results of verification tests using three different values of $L$ $(300 \mathrm{~km}, 500 \mathrm{~km}$, and $700 \mathrm{~km})$. This correlation function is plotted as the dashed line in Figure 15. The proposed new definition of $P_{k j}$ is

$$
P_{k j}=\left(1+\frac{\left|x_{k}-x_{j}\right|}{L}\right) \exp \left[-\frac{\left|x_{k}-x_{j}\right|}{L}\right]
$$

i.e., Curve 3 in equation (21) with $L$ varying as shown Figure 14. This new correlation function is also plotted in Figure 15 for two different values of $L: 200 \mathrm{~km}$ and $500 \mathrm{~km}$. It can be seen that the current operational implementation is closest to the proposed new implementation for $L=200 \mathrm{~km}$. Examination of Figure 14, however, shows that model errors on this small scale do not occur in any geographical region. A more appropriate value for the length scale of the model errors at midlatitudes is $L=500 \mathrm{~km}$.

[54] It is not clear why the earlier assimilation study found that a relatively small value of $L$ provided the best results (i.e., lowest rms error in SWH when compared to in situ buoy observations.) One possible cause for the discrepancy is that the model and observational error variances used may have been inaccurately specified. Another is that the verification sites used by Greenslade [2001] were around the Australian coast and therefore in areas of relatively small $L$. In addition, one of the time periods examined in the earlier study was outside the 4-year time period examined here. It is possible that the error characteristics of the wave model during this alternative time period were different than those found here.

[55] The SWH fields considered in this section are hindcasts; that is, the wave model is forced with winds from the data assimilation cycle of the atmospheric model, but no wave data assimilation is included. Since no ongoing corrections are being made to the wave fields, this means that they are essentially forecasts, albeit forced with analyzed winds. In an operational data assimilation system, the required error correlation length scales and error variances are those which describe the background errors at the time interval at which data are inserted into the model. For the Bureau system, this is 3 hours. The distribution of the background error correlation scales presented in Figure 14 describes errors occurring in longer-range forecasts. So the results found using this method do not exactly represent what is required in an assimilation system.

[56] However, no analysis is perfect: Even with data assimilation, most areas of the ocean are not updated every assimilation period. This is particularly true of wave data assimilation systems that typically use sparse satellite altimeter data. In addition, the length scales and error variances defined in the assimilation system represent expected values and are unlikely to be correct at all times. This means that there will always be some longterm or remotely forced errors in the analysis and thus also in the forecast. The results presented here can be thought of as an upper bound to the error structure of the

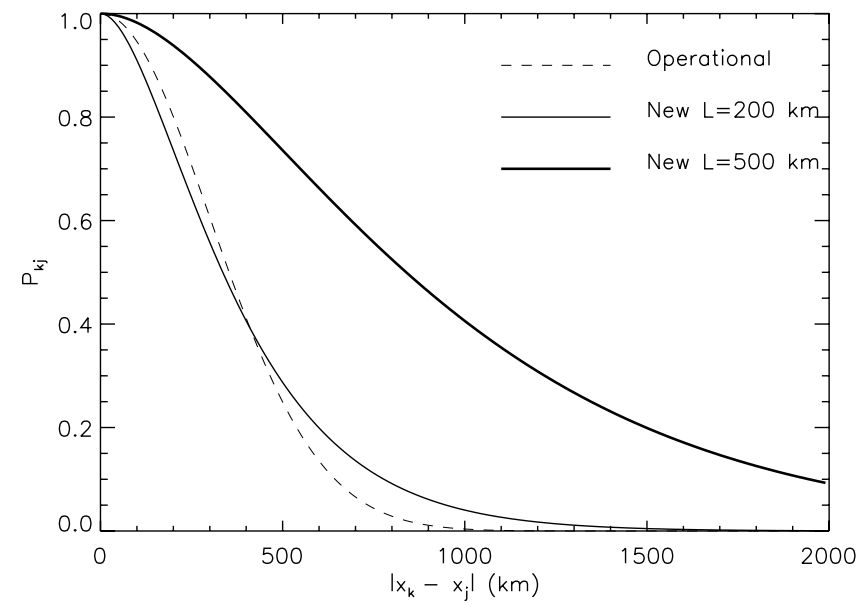

Figure 15. Comparison between current operational model error correlations and results found in this section. 
Jan - Mar

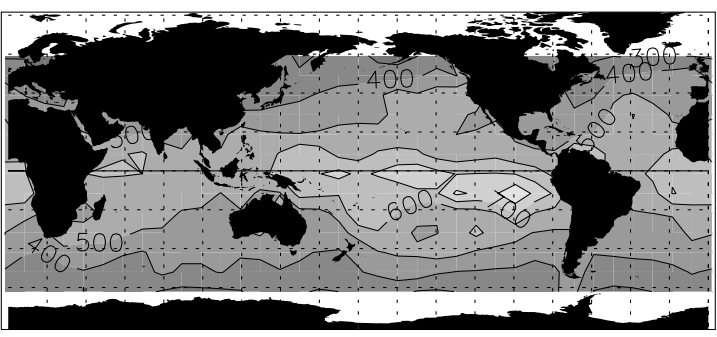

Jul - Sep

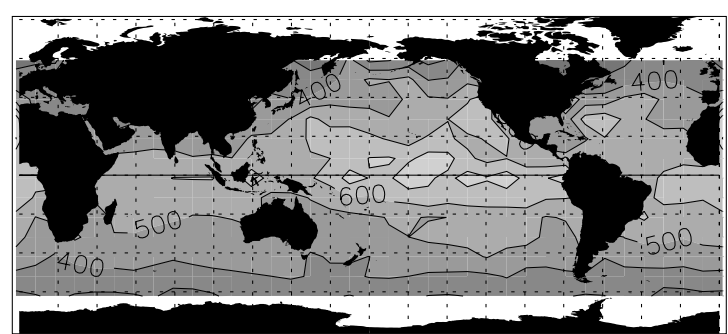

Apr - Jun

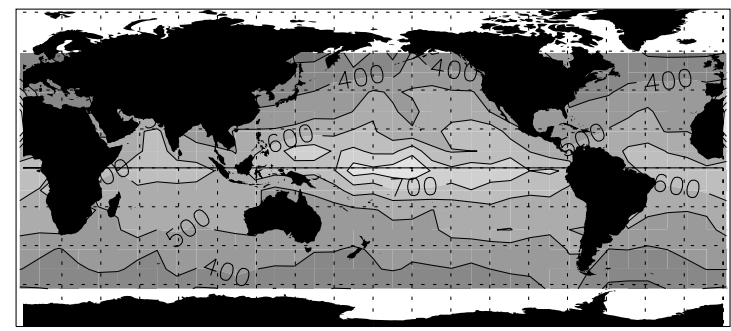

Oct - Dec

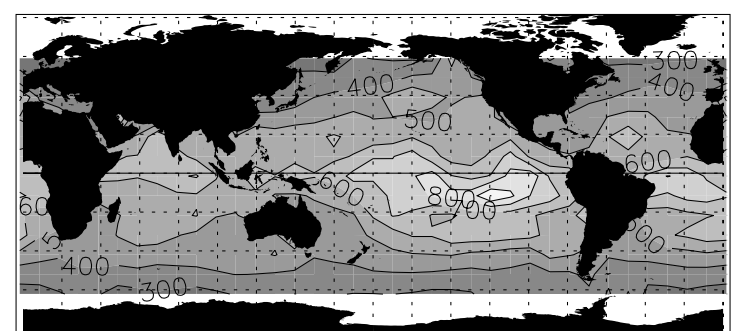

Figure 16. Average model error correlation length scale for each season. These length scales have been adjusted according to equation (25).

background errors. This issue will be addressed in future work by considering the differences between SWH forecasts at different forecast ranges valid at the same time, i.e., by looking at forecast divergence.

[57] There are grounds for expecting seasonal variability in the model error correlations. In ocean models, for example, it is often assumed that the spatial scale of the model errors is similar to the signal or to the climatological anomaly [Oke et al., 2002]. If the model error has the same structure as the model signal, this suggests that in areas where the SWH varies on small spatial scales, the model error would also be expected to vary on small scales. Therefore it might be expected that the region of shortest correlation scales would vary seasonally, shifting to the winter hemisphere where there are more small-scale storms.

[58] The background error length scales for each season are shown in Figure 16, where each panel represents the average over the same season in each of the 4 years. These length scales have been adjusted according to equation (25). The most obvious feature of Figure 16 is that the length scales in the equatorial Pacific are slightly longer during October-December than during the other seasons. However, examination of the length scales for the individual seasons showed that this is due to one particular season (October-December 2000) rather than being a general trend. In general, there is very little seasonal variation in the length scales. Seasonal variability in the background errors of the atmospheric model used to force these modeled wave fields has also been examined. The background errors were investigated via ensemble methods and showed no systematic seasonal variability (R. Seaman, personal communication, 2003). This reinforces the conclusion that there is little seasonal variability in the spatial scale of the wave model errors.

[59] If the models errors are proportional to the climatological anomaly, then one would expect to see similar geographic and seasonal variability as in Figure 8. For example, a signal that stands out strongly in the anomalies is the long length scales in the Indian Ocean during JulySeptember but not January-March. This is associated with the monsoon and is discussed in GY1. In Figure 16, there is no indication of longer length scales in the northern Indian Ocean in the July-September panel of Figure 16 than in any of the other panels. This implies that these large spatial signals occur in both the model and the observations and therefore not the model error. This is further evidence that setting the model error correlations to be proportional to the model anomalies will not always result in accurate estimates of the model errors. Overall, these results suggest that it is not necessary to implement a model error correlation function that varies seasonally.

[60] It is also interesting to examine how the background error correlation length scales vary from year to year. This is shown in Figure 17. One might expect a change in the structure of the model errors when the wind forcing changes characteristics in April 2001. For example, if the wind forcing includes shorter scales, then the total wave model error may include error on smaller scales also. However, as can be seen from Figure 17, there is again very little difference in the spatial scale of the model errors from year to year. This suggests that the increased frequency and resolution of the wind forcing does not have a significant impact on the spatial scale of the errors in the wave model. 
Mar 1998 - Feb 1999

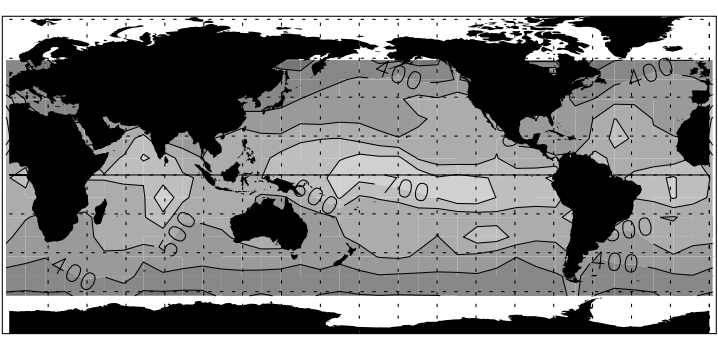

Mar 2000 - Feb 2001

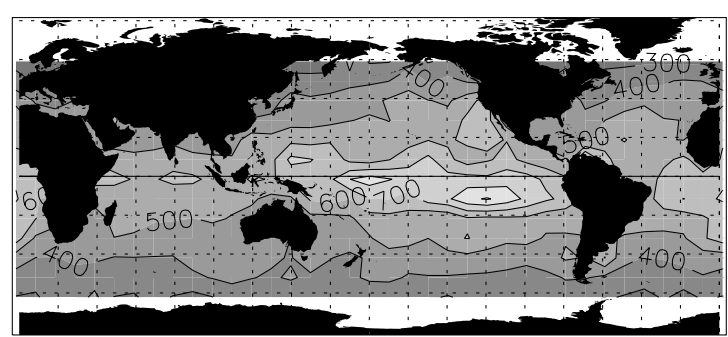

Mar 1999 - Feb 2000

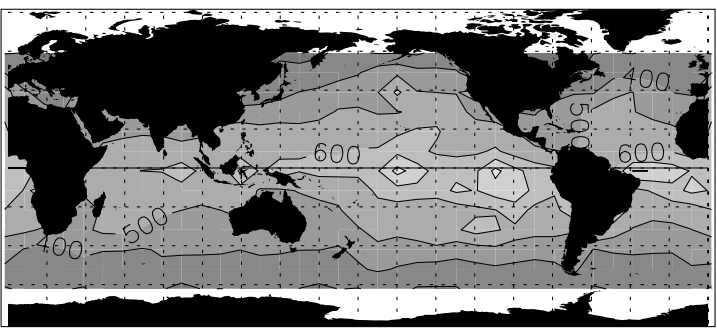

Mar 2001 - Feb 2002

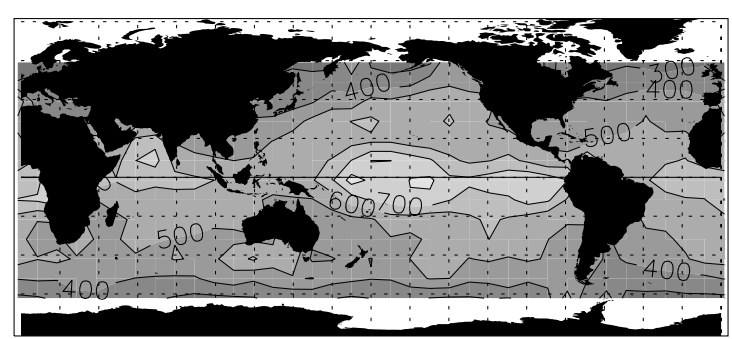

Figure 17. Average model error correlation length scale for each year. These length scales have been adjusted according to equation (25).

[61] The lack of seasonal and year-to-year variability in the correlation length scales, however, may be associated with the fact that the modeled wave fields examined in this work are essentially wave forecasts. The modeled wave fields are not corrected during the 4-year period, so it is likely that there is always a degree of remotely forced and large-scale error at all locations in the wave fields despite the season. It may be that this large-scale swell error dominates the results at all times.

\subsection{Error Variances}

[62] The magnitude of the model and observational errors is now considered. In particular, the model error variance $E_{B}^{2}$ and the observation error variance $E_{O}^{2}$ are estimated. As discussed in section 5.4, equations (18) and (20) can easily be solved for $E_{B}^{2}$ and $E_{O}^{2}$. However, recall that even the bestfit function for Curve 3 often has a large error at the zerointercept (see, for example, Figure 6). This means that the estimate of $R_{0}$ will be inaccurate, resulting in inaccurate estimates of the error variances. A better approximation for $R_{0}$ in equation (18), and the value used here, is the value of the correlation in the 0 to $10 \mathrm{~km}$ bin for each case.

[63] For any particular time period of interest, the method for determining the model and observation error variances is as follows, with step numbers given in parentheses: (1) For each $20^{\circ}$ box, calculate the average correlation over the desired number of seasons. (2) Determine $R_{0}$, the value of the average correlation in the 0 to $10 \mathrm{~km}$ bin. (3) Calculate $E_{B}^{2}+E_{O}^{2}$ according to equation (20). (4) Solve equations (18) and (20) for $E_{B}^{2}$ and $E_{O}^{2}$. The results for the model error variance for the entire four-year time period are shown in Figure 18. The model error variance ranges from less than
$0.2 \mathrm{~m}^{2}$ at low latitudes to around $0.8 \mathrm{~m}^{2}$ in the Southern Ocean with other peaks in the North Pacific and North Atlantic of around $0.7 \mathrm{~m}^{2}$. Comparing this to the mean SWH in Figure 4, it can be seen that the model error variance has a similar global distribution to the $\mathrm{SWH}$, as would be expected. However, the maximum model error variance occurs at the southernmost latitudes, rather than at $50^{\circ} \mathrm{S}$, where $\mathrm{SWH}$ is highest. This is likely to be associated with the fact that the ice edge is not included explicitly in the wave model. Another explanation could be that the altimeter data is of poor quality near the ice edge, but this can be discounted because the observational error variance has been taken into account. Alternatively, the quality of the modeled winds at these southernmost latitudes may be particularly poor.

[64] The error variance of the ERS-2 FD SWH over the 4-year period is shown in Figure 19. It is significantly lower than the model error variance, being less than $0.2 \mathrm{~m}^{2}$ everywhere. Again, there is a latitudinal dependence, with the maximum values of $E_{O}^{2}$ occurring at the highest latitudes. Recall that $E_{O}^{2}$ may be underestimated due to possible spatial correlations in the altimeter observations that have not been taken into account. Further work would be required to quantify this underestimation.

[65] As with the error correlation length scales, the temporal variability of the error variances can be examined. The seasonal variability of the model and ERS-2 error variances are shown in Figures 20 and 21, respectively. As one might expect, the error variance of the model varies quite considerably on a seasonal basis. In general, the pattern follows the pattern of $\mathrm{SWH}$, so that during the Northern Hemisphere winter months (October to March) 


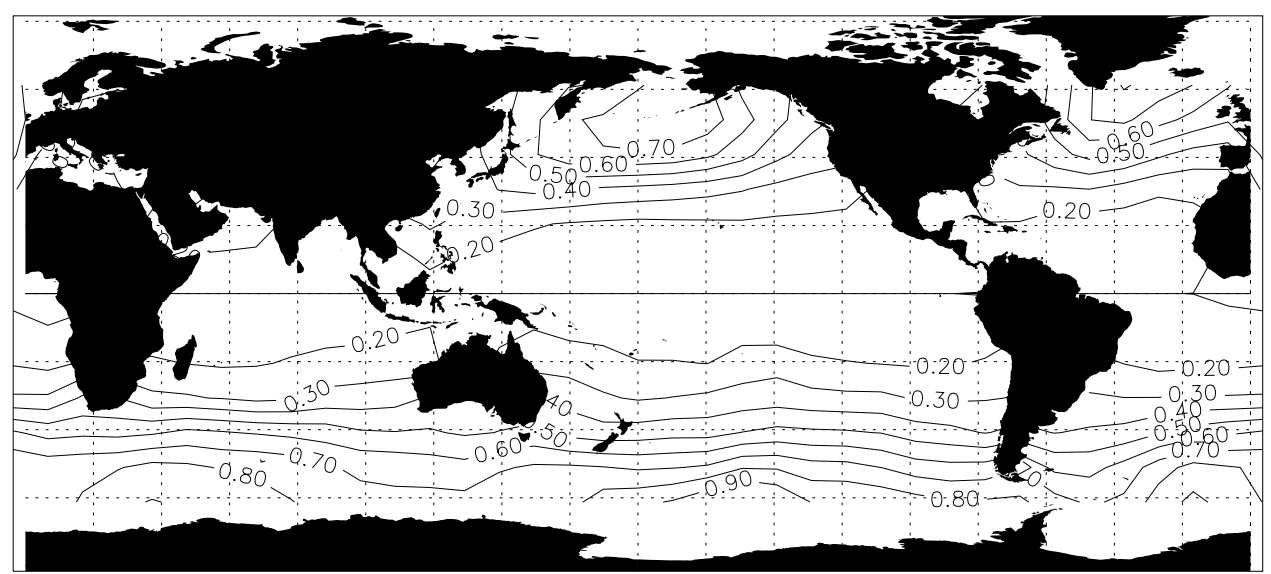

Figure 18. Average model error variance $\left(\mathrm{m}^{2}\right)$ over the entire 4-year period.

the highest error variance is found in the Northern Hemisphere and during the Southern Hemisphere winter months the pattern reverses, with the highest error variance occurring in the Southern Ocean. A similar pattern occurs in the seasonal variability of the ERS-2 error variance. In particular, note that areas where $E_{O}^{2}>0.2 \mathrm{~m}^{2}$ appear only in the winter hemisphere.

[66] The year-to-year variabilities of the model and ERS2 error variances are shown in Figures 22 and 23, respectively. Recall that the length scale of the model error correlations was not found to vary significantly from year to year, despite the change in the characteristics of the wind forcing. Examination of Figure 22 shows that there is a significant reduction in the magnitude of the model error variance during the final year, April 2001 to March 2002. During the first 3 years, the highest $E_{B}^{2}$ in the North Pacific is greater than $0.8 \mathrm{~m}^{2}$, but this is halved in the final year. Similarly, $E_{B}^{2}$ in the Southern Ocean is reduced from $0.8 \mathrm{~m}^{2}$ to $0.6 \mathrm{~m}^{2}$. It was seen in Figure 3 that the changes to the wind forcing fields had a negative impact on the wave model bias. This is probably due to the smoothing in time of the wind fields. It can be seen here that these same changes to the winds have a positive impact on the wave model error variance and this is probably due to the increased frequency and resolution of the wind forcing.
[67] There should not be any year-to-year variability in the ERS-2 error variance, since the observation errors are completely independent of any changes in the model formulation. Figure 23 shows that in general, this is true. In particular, note that the position of the $0.1 \mathrm{~m}^{2}$ contour line in all ocean basins is consistent from year to year.

\subsection{Anisotropic Correlations}

[68] GY1 explored several methods for obtaining anisotropic anomaly correlations from simulated altimeter data. It was concluded that the altimeter ground tracks did not provide enough zonal information to provide realistic anisotropic anomaly correlations. However, it was shown that by considering correlations along ascending and descending ground tracks separately, some indication of the areas on the globe that exhibit the most anisotropy can be obtained.

[69] For each $20^{\circ}$ box and each season, the set of error correlations as a function of distance is divided into two groups: correlations along ascending ground tracks and correlations along descending ground tracks. Correlations from multiple seasons are then averaged together as in section 5. The curve fitting procedure is applied to the ascending and descending groups separately to determine $L_{a s c}$ and $L_{d e s c}$. Only Curve 3 in equation (21) is considered here.

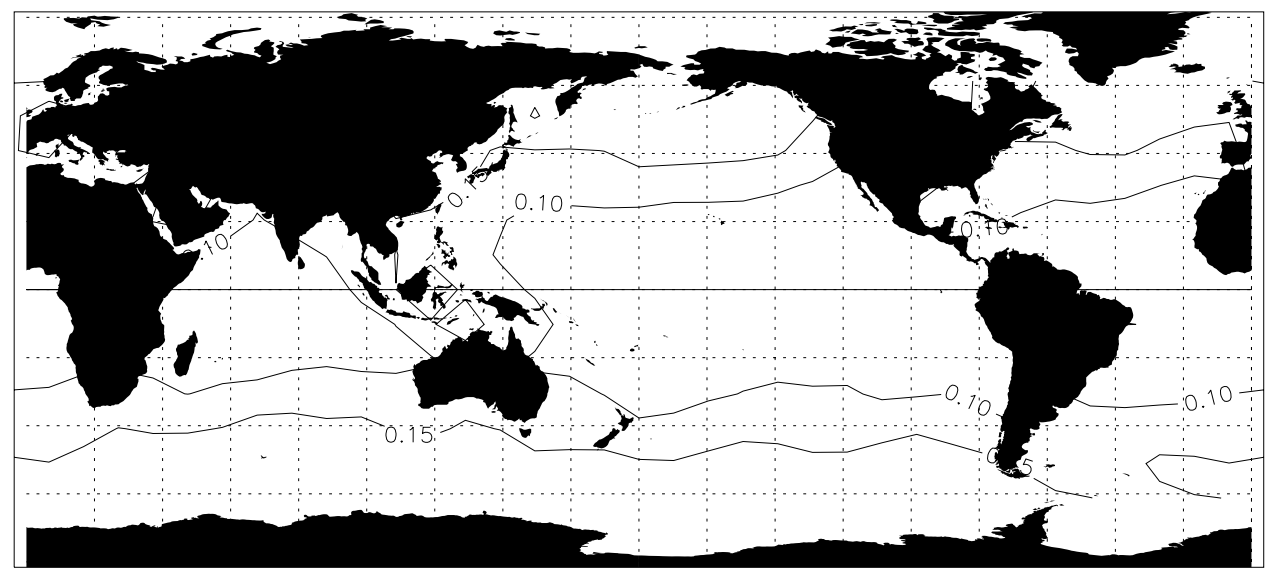

Figure 19. Average ERS-2 error variance $\left(\mathrm{m}^{2}\right)$ over the entire 4-year period. 
Jan - Mar

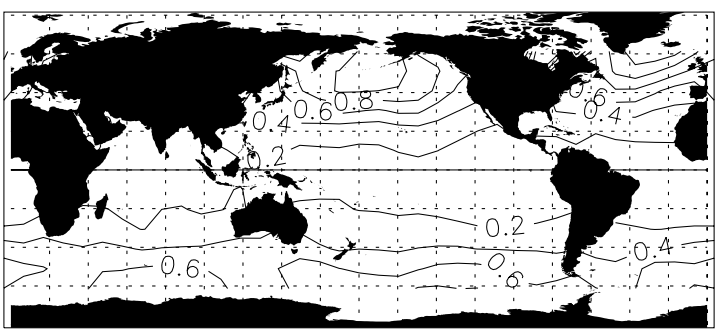

Jul - Sep

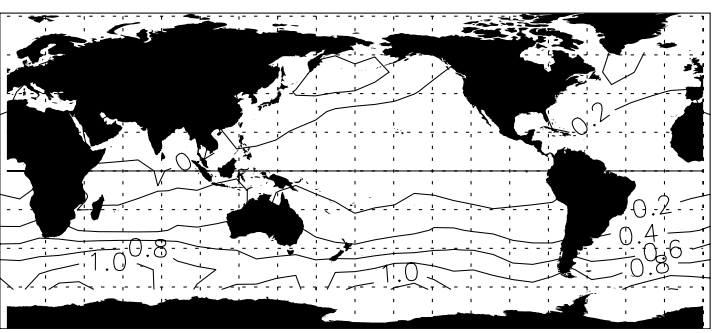

Apr - Jun

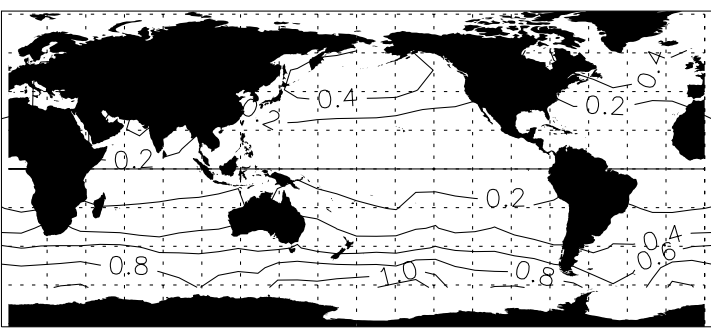

Oct - Dec

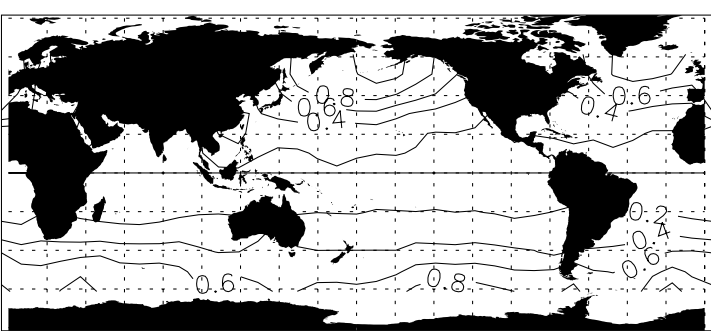

Figure 20. Average model error variance $\left(\mathrm{m}^{2}\right)$ for each season.

[70] The ascending (descending) angle is defined to be the average angle between all data-pairs along ascending (descending) ground tracks. Figure 24 shows an example of the average correlations along ascending and descending ground tracks for the four April to June seasons within a $20^{\circ}$ box in the Indian Ocean. For this particular case, $L_{a s c}=$ $325 \mathrm{~km}$ and $L_{\text {desc }}=190 \mathrm{~km}$. This means that, for example, if the modeled SWH differs from the ERS-2 SWH at a
Jan - Mar

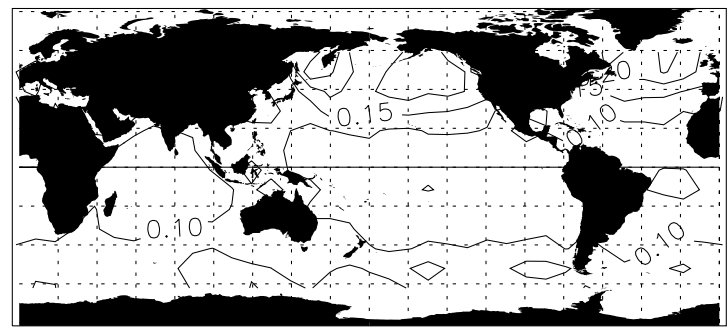

Jul - Sep

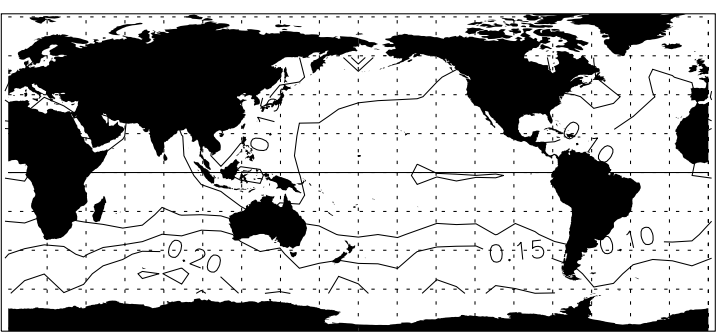

Apr - Jun

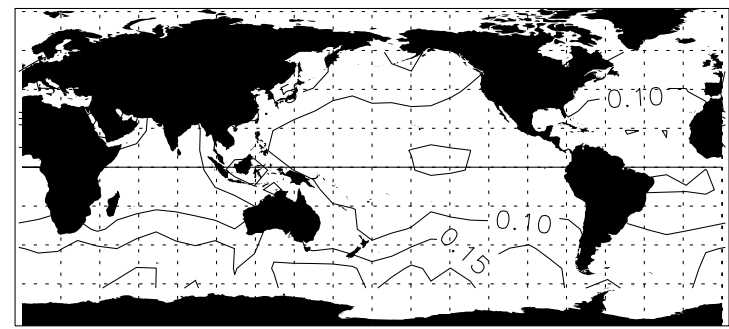

Oct - Dec

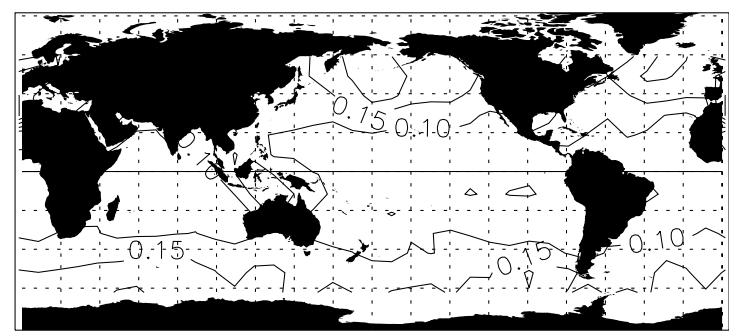

Figure 21. Average ERS-2 error variance $\left(\mathrm{m}^{2}\right)$ for each season. 

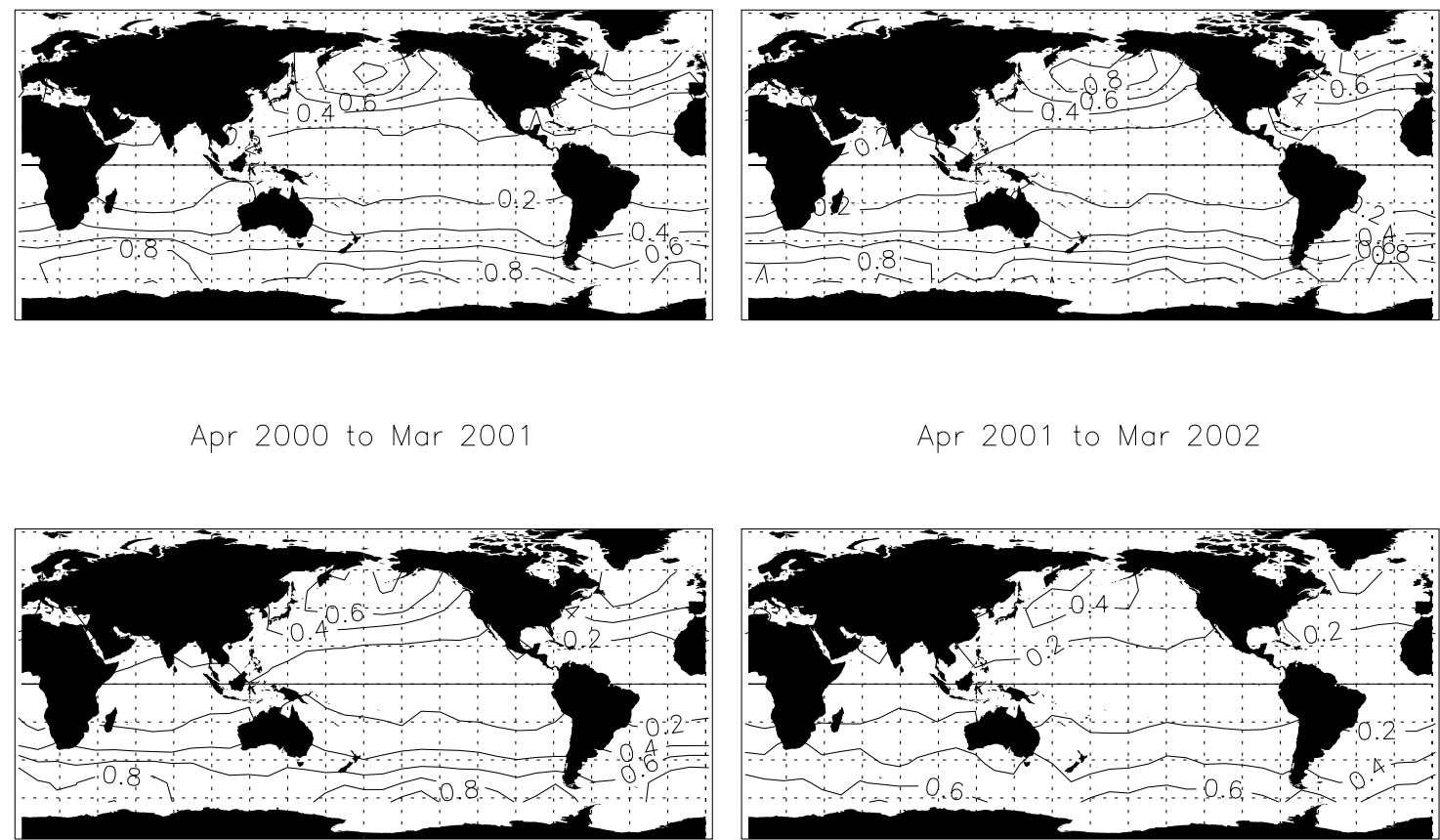

Figure 22. Average model error variance $\left(\mathrm{m}^{2}\right)$ for each year.

particular point within this box, then the modeled SWH at points lying in the direction of the ascending track is more likely to differ from the ERS-2 SWH than points in the direction of the descending track.
[71] An ellipse can easily be constructed given the two length scales and two angles. The major axis of the ellipse must be defined to lie in the direction of either the ascending or descending ground track, which limits the potential
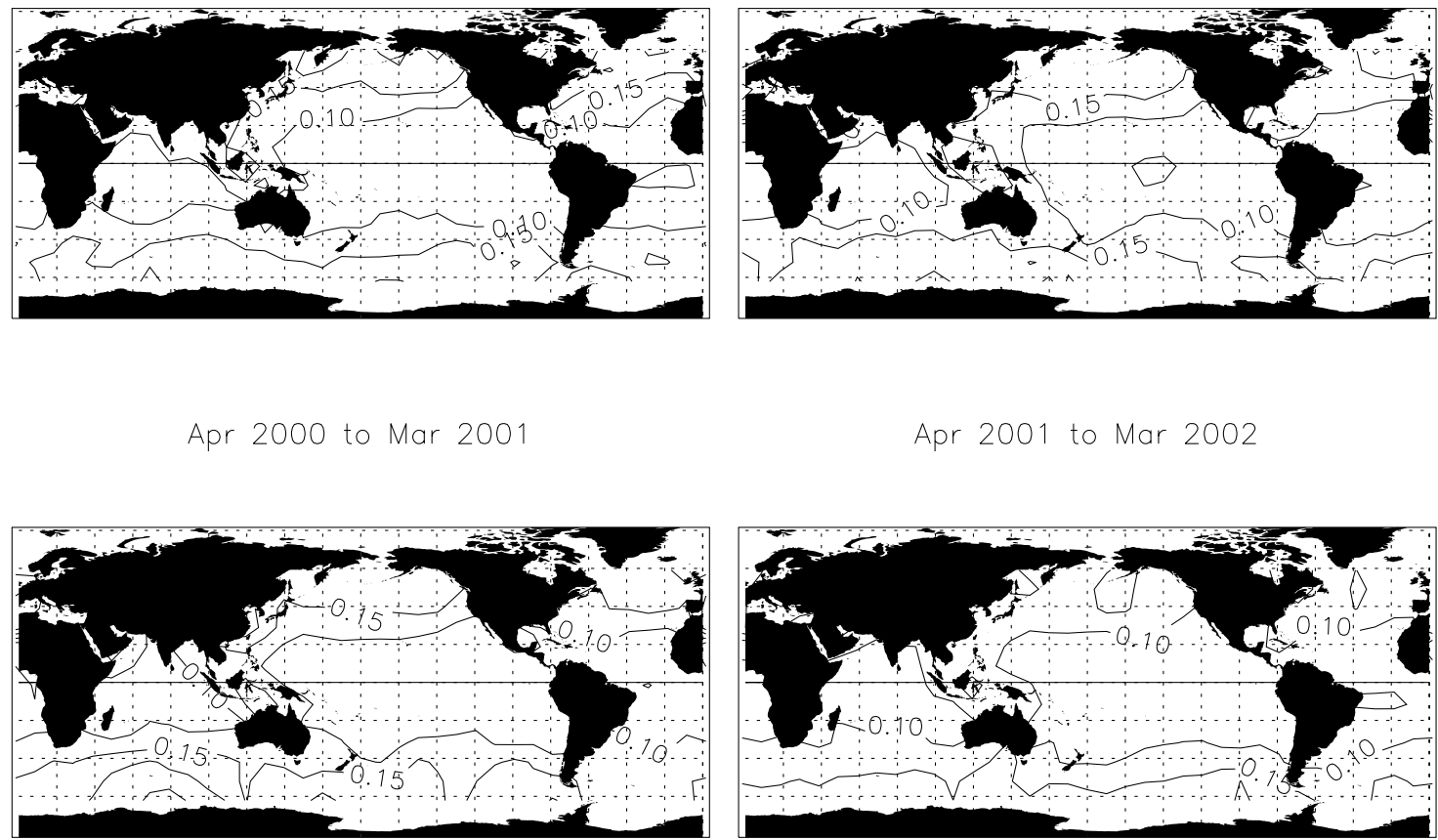

Figure 23. Average ERS-2 error variance $\left(\mathrm{m}^{2}\right)$ for each year. 


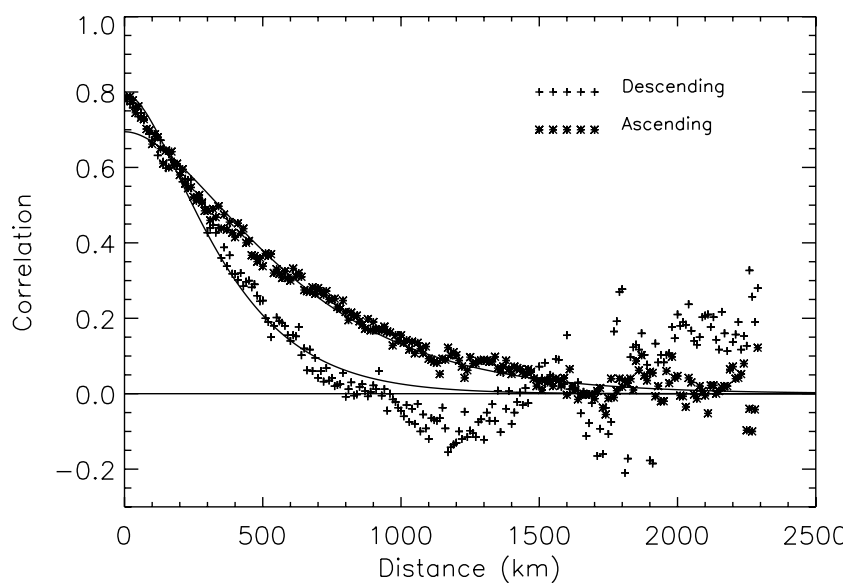

Figure 24. Average correlations along ascending and descending ground tracks within the $20^{\circ}$ box centered at $\left(70^{\circ} \mathrm{E}, 40^{\circ} \mathrm{S}\right)$ for the four April-June seasons.

orientation of the ellipses. Figure 25 shows the ellipses that describe the correlations for all 16 seasons averaged together. Note that the axis length of each ellipse is defined in kilometers and then plotted on the latitude/longitude grid. This means that the differences in the size of the ellipses seen in Figure 25 are real and not simply an artifact of the map projection.

[72] Over most of the ocean, it can be seen that the ellipses are close to circles. This can mean one of two things: either the background errors are generally isotropic or the background errors are anisotropic, but over the 4 years, there is no dominant direction for the longest scales. (Or of course, there is anisotropy but it is aligned in such a way that the altimeter is unable to detect it). To provide a better idea of whether any persistent detectable anisotropy exists in the model errors, the shaded areas in Figure 26 show regions in which one of the length scales (from Figure 25) is at least 25\% larger than the other. It can be seen that over a large portion of the North Atlantic Ocean the model errors are somewhat anisotropic. The most persistently anisotropic area occurs to the south of Australia.
This is likely to have an impact on data assimilation schemes for the Australian region.

\section{Summary}

[73] In this work, background errors of a global wave model have been examined by considering the difference between modeled SWH fields and ERS-2 altimeter observations of SWH over a 4-year period. The ERS-2 observations have been corrected according to a relationship based on colocation with buoy observations. Thus the ERS-2 data can be assumed to be unbiased, and this provides an opportunity to examine the bias in the modeled SWH on a global scale. Changes in the distribution and magnitude of wave model bias were found to coincide with changes in the characteristics of the wind-forcing fields. In general, the largest (negative) biases in SWH were found to occur within a latitude band at $30^{\circ} \mathrm{S}$ to $40^{\circ} \mathrm{S}$. These are associated with known biases in the sea level pressure of the atmospheric model in this region.

[74] Isotropic error correlations for 3-month time periods were calculated over the globe within boxes of side length $20^{\circ}$ in latitude and longitude. The correlations were fit to auto-regressive curves to determine the length scale of the background error. A correction to the length scales that depended linearly on latitude and on the calculated length scale was determined. It was found that the length scale of the background error varies considerably over the globe, with the longest scales at low latitudes and the shortest scales at high latitudes. Very little seasonal or year-to-year variability was detected in the background error length scales.

[75] The magnitude of the model and observational error variances can be estimated with the assumptions that the ERS-2 observations are spatially uncorrelated, and that the background and observational error variances are not correlated with each other. The latter is a reasonable assumption as the ERS-2 data have not been assimilated into the modeled wave fields. The former may contribute to an underestimation of the observational error variance. The average model error variance over the 4-year period was found to range from $0.2 \mathrm{~m}^{2}$ at low latitudes to $0.8 \mathrm{~m}^{2}$ at high latitudes. The error variance in ERS-2 SWH was less than

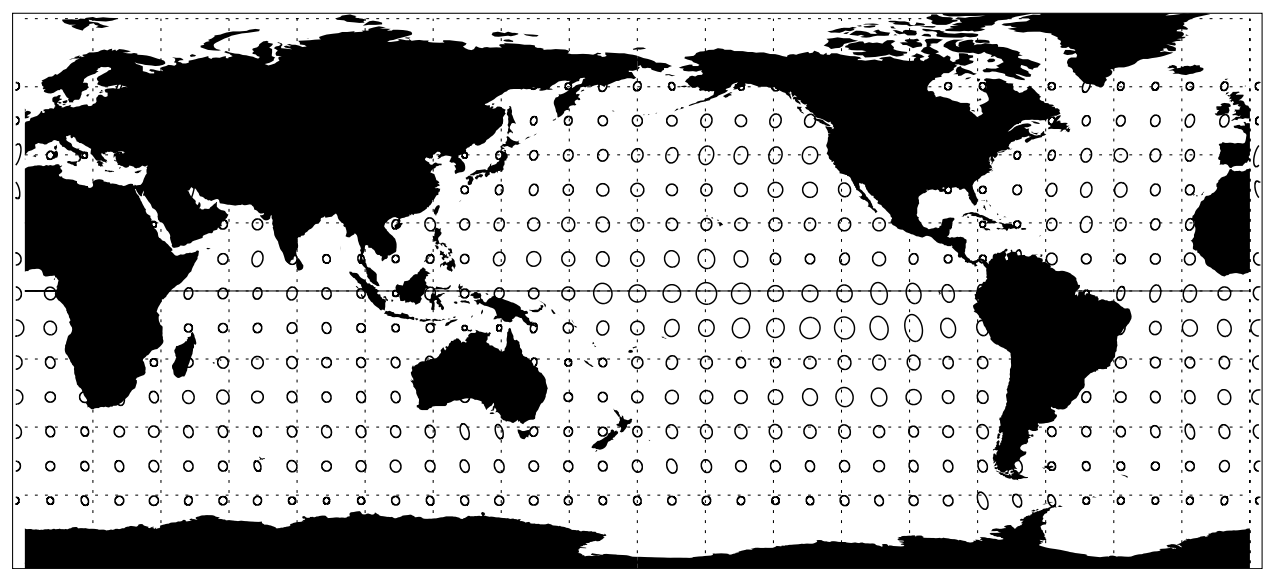

Figure 25. Anisotropic error correlations constructed from ascending and descending ground tracks for all 16 seasons averaged together. 


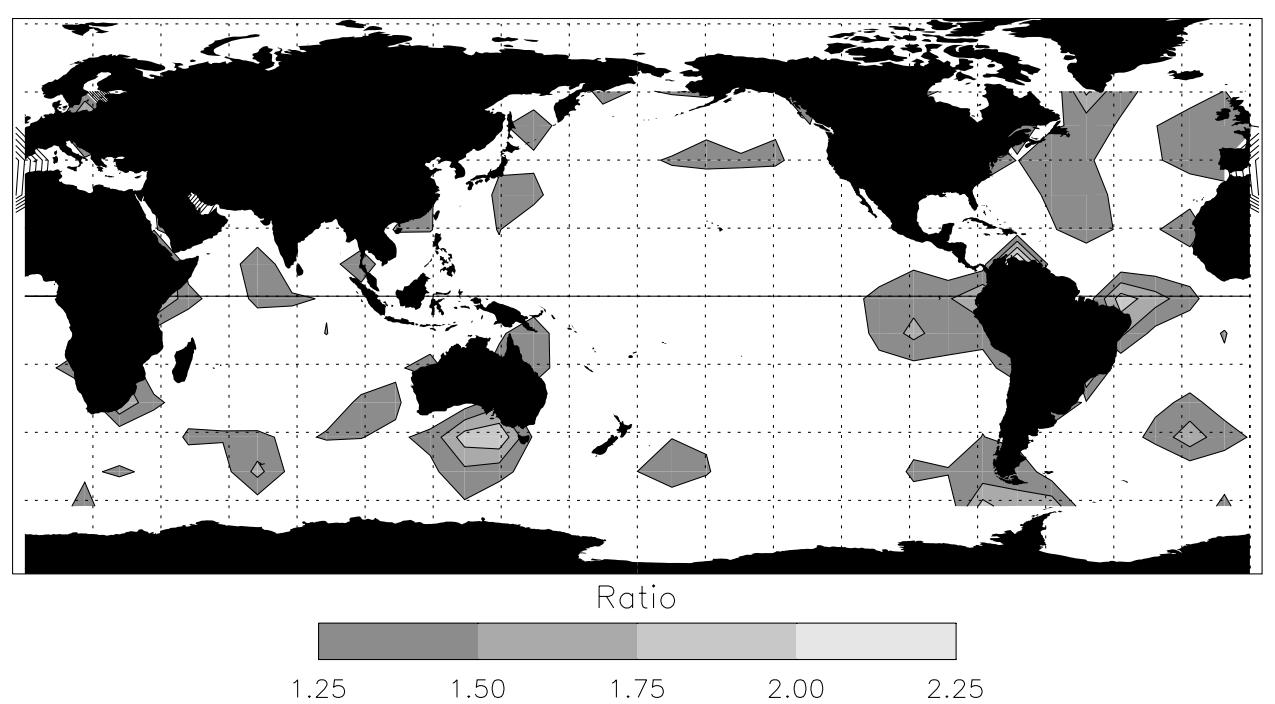

Figure 26. Shaded areas are those in which $L_{\text {asc }}$ is at least $25 \%$ larger than $L_{\text {desc }}$ or vice versa.

$0.2 \mathrm{~m}^{2}$ globally. There was found to be considerable seasonal variability in both the model and observational error variances. In general, the error variances have similar global distributions to that of SWH. There was found to be little year-to-year variability in the observational error variance, but the background error variance decreased substantially with increased frequency and resolution of the wind forcing fields.

[76] The length scales of the error correlations along ascending and descending ERS-2 ground tracks were examined separately. This indicated that anisotropy is likely to be important for the region to the south of Australia and in the North Atlantic Ocean.

[77] One issue that has been raised in this work is that the modeled wave fields used are essentially model forecasts, albeit forced with "analyzed" winds. In an operational data assimilation system, the required description of the background errors is that which describes the errors at the time interval at which data is inserted into the model. This means that the results found using this method do not exactly represent what is required in an assimilation system. However, even with data assimilation most areas of the ocean are not updated every assimilation period. This is particularly true of the sparse altimeter data that is typically used in wave data assimilation systems. This means that there will always be some long-term or remotely forced errors in the analysis and thus also in the forecast. The results presented here can be thought of as an upper bound to the error structure of the background errors and error variance.

[78] In future work, this issue will be addressed by considering the differences between SWH forecasts at different forecast ranges valid at the same time, i.e., by looking at forecast divergence. This method may also provide more information on the anisotropy of the model errors. Other future work involves examining the impact of the proposed new prediction error correlation matrix on the forecast skill of a wave data assimilation system.

[79] Acknowledgments. The authors would like to thank Peter Steinle and Bob Seaman for reviewing early drafts of the manuscript.
Financial assistance in the form of a Ph.D. scholarship for D. J. M. Greenslade was provided by the Australian Bureau of Meteorology.

\section{References}

Bender, L. C. (1996), Modification of the physics and numerics in a thirdgeneration ocean wave model, J. Atmos. Oceanic Technol., 13, 726-750.

Bender, L. C., and T. Glowacki (1996), The assimilation of altimeter data into the Australian wave model, Aust. Meteorol. Mag., 45, 41-48.

Bender, L. C., and L. M. Leslie (1994), Evaluation of a third generation ocean wave model for the Australian region, BMRC Res. Rep. 43, Bureau of Meteorol., Melbourne, Australia.

Bidlot, J. R., P. A. E. M. Janssen, B. Hansen, and H. Günther (1997), A modified set up of the advection scheme in the ECMWF wave model, ECMWF Tech. Memo. 237, Eur. Cent. for Medium-Range Weather Forecast., Reading, England.

Breivik, L. A., and M. Reistad (1994), Assimilation of ERS-1 altimeter wave heights in an operational numerical wave model, Weather Forecast., 9, 440-451.

Breivik, L. A., M. Reistad, H. Schyberg, J. Sunde, H. E. Krogstad, and H. Johnsen (1998), Assimilation of ERS SAR wave spectra in an operational wave model, J. Geophys. Res., 103, 7887-7900.

Daley, R. (1991), Atmospheric Data Analysis, 457 pp., Cambridge Univ. Press, New York.

Dunlap, E. M., R. B. Olsen, L. Wilson, S. De Margerie, and R. Lalbeharry (1998), The effect of assimilating ERS-1 fast delivery wave data into the North Atlantic WAM model, J. Geophys. Res., 103, 7901-7915.

Garratt, J. R. (1992), The Atmospheric Boundary Layer, 316 pp., Cambridge Univ. Press, New York.

Greenslade, D. J. M. (2001), The assimilation of ERS-2 significant wave height data in the Australian region, J. Mar. Syst., 28, 141-160.

Greenslade, D. J. M., and I. R. Young (2004), A validation of ERS-2 fast delivery significant wave height, BMRC Res. Rep. 97, Bureau of Meteorol., Melbourne, Australia.

Hasselmann, S., P. Lionello, and K. Hasselmann (1997), An optimal interpolation scheme for the assimilation of spectral wave data, J. Geophys. Res., 102, 15,823-15,836.

Hollingsworth, A., and P. Lönnberg (1986), The statistical structure of short-range forecast errors as determined from radiosonde data: I. The wind field, Tellus, Ser. A, 38, 111-136.

Janssen, P. A. E. M. (1989), Wave-induced stress and the drag of air flow over sea waves, J. Phys. Oceanogr., 19, 645-754.

Janssen, P. A. E. M. (1991), Quasi-linear theory of wind-wave generation applied to wave forecasting, J. Phys. Oceanogr, 21, 1631-1642.

Janssen, P. A. E. M., S. Abdalla, and H. Hersbach (2003), Error estimation of buoy, satellite and model wave height data, ECMWF Tech. Memo. 402, Eur. Cent. for Medium-Range Weather Forecast., Reading, England.

Julian, P., and H. J. Thiébaux (1975), On some properties of correlation functions used in optimum interpolation schemes, Mon. Weather Rev., $103,605-616$.

Kepert, J. D., D. J. M. Greenslade, and D. Hess (2004), Assessing and improving the marine surface winds in the Bureau of Meteorology Numerical Weather Prediction Systems, BMRC Res. Rep., in press. 
Komen, G. J., S. Hasselmann, and K. Hasselmann (1984), On the existence of a fully developed wind-sea spectrum, J. Phys. Oceanogr., 14, 12711285.

Komen, G. J., L. Cavaleri, M. Donelan, K. Hasselmann, S. Hasselmann, and P. A. E. M. Janssen (1994), in Dynamic Modelling of Ocean Waves, 532 pp., Cambridge Univ. Press, New York.

Lionello, P., H. Günther, and P. A. E. M. Janssen (1992), Assimilation of altimeter data in a global third-generation wave model, J. Geophys. Res., 97, 14,453-14,474.

Lönnberg, P., and A. Hollingsworth (1986), The statistical structure of short-range forecast errors as determined from radiosonde data: II. The covariance of height and wind errors, Tellus, Ser. A, 38, 137161.

Lorenc, A. C. (1981), A global three-dimensional multivariate statistical interpolation scheme, Mon. Weather Rev., 109, 701-721.

Mastenbroek, C., V. K. Makin, A. C. Voorrips, and G. J. Komen (1994), Validation of ERS-1 altimeter wave height measurements and assimilation in a North Sea wave model, Global Atmos. Ocean Syst., 2, $143-$ 161.

National Meteorological Operations Centre (1998), Upgrade of the Global Analysis and Prediction (GASP) system, Op. Bull. 45, Bureau of Meteorol., Melbourne, Australia.

National Meteorological Operations Centre (1999), Changes to the Operational Sea State Forecast System, Op. Bull. 47, Bureau of Meteorol., Melbourne, Australia.

National Meteorological Operations Centre (2003), Analysis and prediction quarterly summary, April-June 2003, report, Bureau of Meteorol., Melbourne, Australia.

Oke, P. R., J. S. Allen, R. N. Miller, G. D. Egbert, and P. K. Kosro (2002) Assimilation of surface velocity data into a primitive equation coasta ocean model, J. Geophys. Res., 107(C9), 3122, doi:10.1029/ 2000JC000511
Seaman, R. S. (1982), A systematic description of the spatial variability of geopotential and temperature in the Australian region, Aust. Meteorol. Mag., 30, 133-141.

Seaman, R., W. Bourke, P. Steinle, T. Hart, G. Embery, M. Naughton, and L. Rikus (1995), Evolution of the Bureau of Meteorology's Global Assimilation and Prediction System: 1. Analyses and initialization, Aust. Meteorol. Mag., 44, 1-18.

Snyder, R. L., F. W. Dobson, J. A. Elliot, and R. B. Long (1981), Array measurements of atmospheric pressure fluctuations above surface gravity waves, J. Fluid. Mech., 102, 1-59.

Thiébaux, H. J., H. L. Mitchell, and D. W. Shantz (1986), Horizontal structure of hemispheric forecast error correlations for geopotential and temperature, Mon. Weather Rev., 114, 1048-1066.

Tolman, H. L. (1998), Validation of NCEP's ocean winds for the use in wind wave models, Global Atmos. Ocean Syst., 6, 243-268.

Voorrips, A. C., K. Makin, and S. Hasselmann (1997), Assimilation of wave spectra from pitch-and-roll buoys in a North Sea wave model, J. Geophys. Res., 102, 5829-5849.

WAMDI Group (1988), The WAM model - A third generation wave prediction model, J. Phys. Oceanogr., 18, 1775-1810.

Young, I. R. (1999), Wind-Generated Ocean Waves, 288 pp., Elsevier Sci., New York

Young, I. R., and T. J. Glowacki (1996), Assimilation of altimeter wave height data into a spectral wave model using statistical interpolation, Ocean Eng., 23(8), 667-689.

D. J. M. Greenslade, Bureau of Meteorology Research Centre, GPO Box 1289K, Melbourne, Victoria 3001, Australia. (dag@bom.gov.au)

I. R. Young, Swinburne University of Technology, P. O. Box 218 , Hawthorn, Melbourne, Victoria 3122, Australia. (iyoung@swin.edu.au) 